\title{
Spatial Distribution of the Net Burgers Vector Density in a Deformed Single Crystal
}

\author{
A. Sarac*, M.S. Oztop* ${ }^{*}$ C.F.O. Dahlberg ${ }^{*}$ and J.W. Kysar ${ }^{*}$ \\ *Department of Mechanical Engineering, Columbia University, New York, NY \\ 10027
}

June 29, 2016

\begin{abstract}
A two-dimensional deformation field on an indented single crystal, where the only nonzero lattice rotation occurs in the plane of deformation and only three effective in-plane slip systems are activated, is investigated both experimentally and numerically. Electron Backscatter Diffraction (EBSD) is utilized to probe the lattice rotation field on the sample. The lattice rotation field is utilized to calculate the two non-zero components of Nye's dislocation density tensor, which serves as a link between plastic and elastic deformation states. The enhanced accuracy of EBSD enabled measurements of the net Burgers vector density, and a new quantity $\beta$, which monitors the activity of slip systems in the deformed zone. The $\beta$-field is compared to the slip system activity obtained by analytical solution and also by crystal plasticity simulations. A qualitative comparison of the three methods confirms that the $\beta$-field obtained experimentally agrees with the slip system activity obtained analytically and by numerical methods.
\end{abstract}

Keywords: $\beta$-variable; $\beta$-fields; Single Crystal; Lattice Rotation; Electron Backscatter Diffraction (EBSD); Net Burgers Vector Density; Nye's Dislocation Density Tensor; Hardening Parameters; Latent Hardening Ratio 


\section{Introduction}

The Burgers vector, $\mathbf{b}$, is a fundamental quantity in the study of dislocation-mediated plastic deformation that denotes the magnitude and direction of discrete atomic-scale plastic slip events on a slip plane with unit normal vector $\mathbf{n}$ due to the motion of a single dislocation. As illustrated in Fig. 1(a) and (b), the Burgers vector is determined by taking a Burgers circuit on the crystal lattice around an area pierced by an isolated dislocation line. Sustained plastic deformation requires the creation of a great many dislocations that move and ultimately come to rest in the crystal lattice. The final dislocation content is a signature of the plastic deformation that created it. As illustrated in Fig. 1(c) and (d), a Burgers circuit to characterize the final dislocation content will, in general, contain many dislocations from several different slip systems. The closure failure vector, denoted as $\mathbf{b}_{n e t}^{p}$, is the sum of the Burgers vectors piercing the circuit. The net Burgers vector density is the normalization of the closure failure vector by the area $A$, which is bounded by $\Gamma$ as illustrated in Fig. 1.

The geometric relations in crystalline solids with line defects have been studied by means of a dislocation density tensor. This revealed a direct connexion between the elastic and plastic portions of the deformation gradient tensor (Nye, 1953). The dislocation density tensor (Nye, 1953; Kröner, 1958; Bilby et al., 1958), also referred to as Nye's dislocation density tensor, is a second-rank tensor, and determines the state of dislocation in a deformed crystal. It can also be defined as a linear transformation of a unit tangent vector to the net Burgers vector density. The geometric relations defined by Nye (1953) relate to the lattice curvature calculations, and to the geometrically necessary dislocations that are necessary to form a lattice curvature. The geometrically necessary dislocations (GNDs) contribute to Nye's dislocation density tensor, and can be estimated using some minimization scheme (Arsenlis \& Parks, 1999; Kysar et al., 2010). The rest of the dislocations, which make no contribution to Nye's dislocation density tensor, are called the statistically-stored dislocations (SSDs). SSDs accumulate in single crystals during a plastic deformation process (Ashby, 1970).

Nye's dislocation density tensor can be measured from the elastic portion of the deformation gradient tensor, which is associated with the lattice rotation and strain of the crystal lattice. The gradient of the lattice rotation measurements about the three perpendicular axes gives the lattice curvature tensor. There are several ways to accomplish the measuring of lattice rotations. The common method is to measure the orientations of the crystal lattice using Orientation Imaging Microscopy (OIM) (Adams et al., 1993; Adams, 1997), also known as Electron Backscatter Diffraction (EBSD). The method essentially employs a fully-automated scanning technology and EBSD-patterns to obtain 2D-images of lattice distortions (Gardner et al., 2010). Sun et al. (2000) reported the lattice curvature patterns of a plastically deformed high purity Al bicrystal using lattice orientations measured by conventional EBSD. El-Dasher et al. (2003) also measured the lattice curvature through conventional EBSD to estimate GNDs, and discussed the principal limitations of the conventional EBSD method. Orientation gradients of deformed Al single crystals and polycrystals have been measured by other researchers (Field et al., 2005 and Pantleon, 2008) using this method. Pantleon (2008) addressed the limitation of the conventional EBSD method in which there is no possibility to measure the curvatures along the third direction. Beausir \& Fressengeas (2013) used EBSD

orientation mapping to quantify rotational defects in polycrystalline materials. To eliminate 
the disadvantages associated with angular and spatial resolution, High Resolution Electron Backscatter Diffraction (HR-EBSD) has been used by researchers to measure elastic strains, lattice rotations and GNDs (Britton \& Wilkinson, 2011, Britton \& Wilkinson, 2012; Maurice et al., 2012; Ruggles \& Fullwood, 2013; Jiang et al., 2015). The problem of quantifying the out-of-plane components of the elastic deformation gradient tensor can be eliminated using different methods. The first method is to use X-ray microbeam diffraction method to measure lattice rotation and lattice strains (Larson et al., 2004; Ice et al., 2005; Larson et al., 2007; Ohashi et al., 2009; Hofmann et al., 2013; Pang et al., 2014). With X-ray microbeam diffraction, it is possible to probe GNDs and lattice distortions much deeper in the sample. A comprehensive study has been performed by Field et al. (2010) to compare the strain measurements in deformed crystals using both EBSD and X-ray microbeam diffraction. The second method is to use EBSD or Transmission Electron Microscopy (TEM) to measure lattice rotation fields via serial sectioning of the sample by Focused Ion Beam (FIB)(Kiener et al., 2006; Zaafarani et al., 2006; Rester et al., 2007; Demir et al., 2009). The third method is to introduce a two-dimensional plane strain deformation state where all out-of-plane lattice rotations are negligible, in other words, the nonzero components of the lattice curvature tensor can only be obtained from the in-plane lattice rotations (Kysar \& Briant, 2002; Gan et al., 2006; Kysar et al., 2007; Kysar et al., 2010).

The objective of the present study is to define a new variable, $\beta$, for validation of existing elastic-plastic constitutive models and obtain direct information about the state of the material (N.B. In the present study, the authors will not suggest a new constitutive solution, and will not extend the existing constitutive models in any way with the use of the $\beta$-variable defined herein). To do so, a Scanning Electron Microscopy (SEM) and EBSD method was used to measure in-plane lattice rotations. The gradient of in-plane lattice rotation field gives the non-zero components of the lattice curvature tensor, which correspond to non-zero components of Nye's dislocation density tensor. Nye's dislocation density tensor, which is a linear transformation of a unit tangent line vector to the net Burgers vector density, serves as a link between elastic and plastic deformation. The orientation of the net Burgers vector density determines the newly defined $\beta$-variable. The contour maps of the spatially resolved $\beta$-variables are called the $\beta$-fields, which quantify the spatially resolved defect densities for a material in its deformed state. The $\beta$-fields are also utilized to determine and characterize the slip system activities. To analyze the effects of the single crystal hardening rules on the $\beta$-fields, Finite Element Simulations pertaining to two different hardening models were performed. The effects of the hardening parameters on the $\beta$-field were analyzed, and experimental data was used as a benchmark.

The paper is organized as follows. The theoretical background of the net Burgers vector density including the calculation of its magnitude and orientation is discussed in Section 2. Deformed and undeformed configurations of the specimen and the experimental procedure are outlined in Section 3. The differences between the crystallographic and effective in-plane slip systems are also given in this section. The finite element model with relevant parameters is briefly described in Section 4. The hardening parameters associated with the hardening models are defined and listed in Subsection 4.2. The $\beta$-fields pertaining to the experiment and Finite Element Simulations are presented and discussed in Section 5. Concluding remarks are given in Section 6 . 
a)

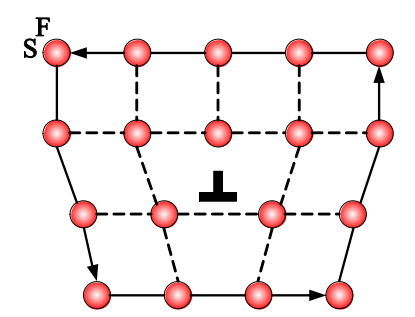

c)

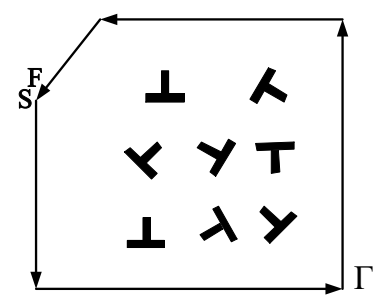

b)

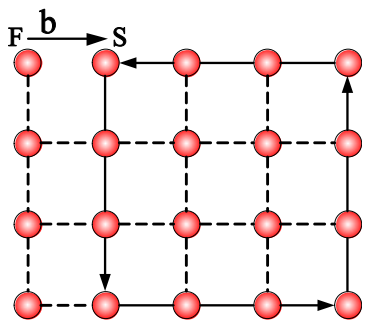

d)

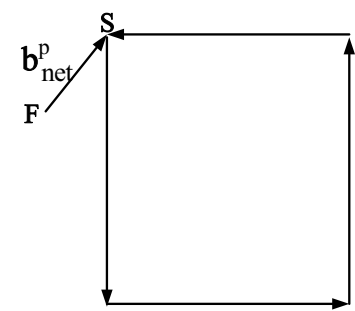

Figure 1: Crystal lattice and the Burgers circuits: (a) Crystal lattice with a single dislocation, (b) the Burgers vector, b, (c) Crystal body with multiple dislocations (d) the net Burgers vector, $\mathbf{b}_{n e t}^{p}$.

\section{Theoretical Background}

The net Burgers vector density, also referred to as the net closure failure density, is measurable via discrete methods (i.e. TEM) or continuum methods (i.e. SEM and EBSD). The net Burgers vector density can be quantified as

$$
\mathbf{B}=\alpha \cdot \ell,
$$

where $\alpha$ is Nye's tensor (also called dislocation density tensor), and $\boldsymbol{\ell}$ is the out-of-plane unit normal vector when the Burgers circuit, $\Gamma$, is taken in a right-hand sense. The dislocation density tensor is a measure of incompatibility serving as a link between the elastic deformation and plastic deformation of the crystal. It can be expressed in terms of the elastic deformation by

$$
\alpha_{j i}=-\kappa_{i j}+\delta_{i j} \kappa_{k k}+e_{i p k} \epsilon_{j k, p}^{e l},
$$

where $\kappa_{i j}$ is the lattice curvature tensor, $\delta_{i j}$ is the Kronecker delta, $e_{i p k}$ is the permutation tensor, $\epsilon_{j k}^{e l}$ is the elastic strain of the crystal lattice, and $\epsilon_{j k, p}^{e l}$ is the elastic strain gradient. The magnitude of the components of the elastic strain gradient is significantly less than the magnitude of the lattice curvatures in Eq. (2). As a result, the elastic strain gradient is negligible by considering a plane strain deformation state and finite deformation kinematics (Kysar et al., 2010). The lattice curvatures are basically the lattice rotation gradients which can be calculated via numerical differentiation of the lattice rotation measurements with respect to the global or reference coordinate frame, and can be denoted as

$$
\kappa_{i j}=\frac{\partial \omega_{i}}{\partial x_{j}} \quad i, j=1,2,3
$$


The lattice rotations about $x_{1}-, x_{2}-$, and $x_{3}$-axes are represented by $\omega_{1}, \omega_{2}$, and $\omega_{3}$, respectively. For the plane strain deformation state we assume approximately that out-ofplane lattice rotations, $\omega_{1}$ and $\omega_{2}$, are negligible compared to $\omega_{3}$, that is $\omega_{1}=\omega_{2}=0$. The nonzero components of the lattice curvature tensor are obtained from the in-plane lattice rotation, $\omega_{3}$. The matrix representation of the non-symmetric lattice curvature tensor is

$$
\kappa=\left[\begin{array}{ccc}
0 & 0 & 0 \\
0 & 0 & 0 \\
\kappa_{31} & \kappa_{32} & 0
\end{array}\right],
$$

where $\kappa_{31}=\frac{\partial \omega_{3}}{\partial x_{1}}$ and $\kappa_{32}=\frac{\partial \omega_{3}}{\partial x_{2}}$, respectively. The trace of the lattice curvature tensor, $\kappa_{k k}$, is zero. By considering the plane strain deformation state, Eq. (2), which is also called the elastic incompatibility equation, can be reduced to Eq. (5) expressed as

$$
\alpha_{j i} \approx-\kappa_{i j} \text {. }
$$

Consequently, the non-zero components of the dislocation density tensor in the reference frame are $\alpha_{13} \approx-\kappa_{31}$ and $\alpha_{23} \approx-\kappa_{32}$. These components are measurable through continuum methods. By applying a coordinate transformation to Eq. (5) one can obtain the Nye's dislocation density tensor in a local crystallographic coordinate frame as

$$
\alpha_{i j}^{\prime}=m_{i k} m_{j l} \alpha_{k l},
$$

where $\alpha_{k l}$ is the Nye's dislocation density tensor in the reference coordinate frame, and $m_{i j}$ is the coordinate transformation tensor which has the following matrix representation

$$
m=\left[\begin{array}{ccc}
\cos \omega_{3} & \sin \omega_{3} & 0 \\
-\sin \omega_{3} & \cos \omega_{3} & 0 \\
0 & 0 & 1
\end{array}\right] .
$$

The magnitude and the direction of the net Burgers vector density, $\mathbf{B}$, can be determined by Eq. (1) in the local crystallographic coordinate frame, where the out-of-plane unit normal vector is in the direction of $x_{3}^{\prime}$-axis, that is $\boldsymbol{\ell}=\hat{e}_{3}^{\prime}$. By substituting Eq. (5, 6 and 7$)$ and $\boldsymbol{\ell}$ back into the Eq. (1), the net Burgers vector density can be quantified as

$$
\mathbf{B}=\alpha_{13}^{\prime} \hat{\mathbf{e}}_{\mathbf{1}}^{\prime}+\alpha_{23}^{\prime} \hat{\mathbf{e}}_{\mathbf{2}}^{\prime} \text {. }
$$

Hence, the magnitude of the net Burgers vector density can be calculated as $B=$ $\sqrt{\left(\alpha_{13}^{\prime}\right)^{2}+\left(\alpha_{23}^{\prime}\right)^{2}}$ in units of length/area or $1 /$ lengths. The orientation angle of the net Burgers vector density can be defined as

$$
\beta=\arctan \left(\frac{\alpha_{23}^{\prime}}{\alpha_{13}^{\prime}}\right) .
$$

This quantity is called the $\beta$-variable, and the spatial distribution of the $\beta$-variables over the deformed surface is referred to as the $\beta$-field. 


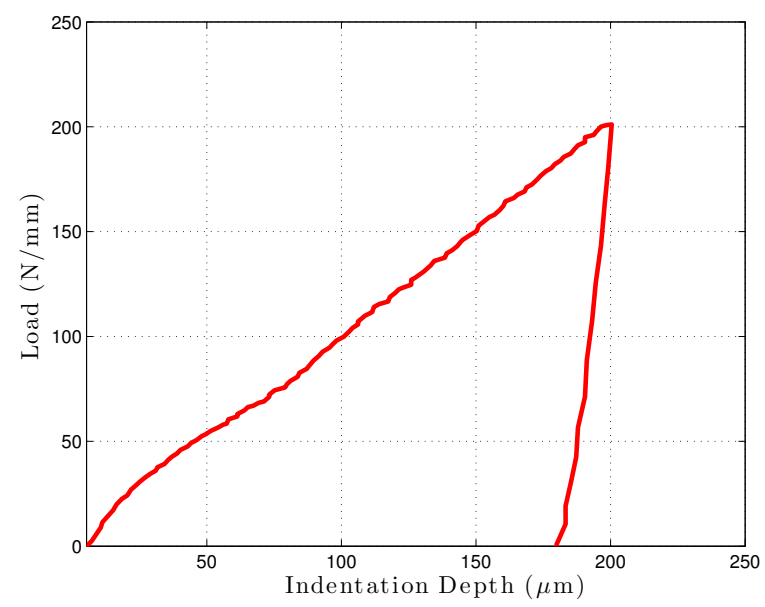

Figure 2: Force as a function of displacement.

\section{$3 \quad$ Experimental Methods and Results}

Experimental Methods are mainly composed of two parts. The first part is the preparation of the single crystal specimen and indentation process. The second part is the EBSD measurements to obtain in-plane lattice rotations and the net Burgers vector density. A filtering process is applied to the spatially resolved net Burgers vector density.

\subsection{Single Crystal Specimen and Indentation}

A high purity rectangular parallelepiped nickel single crystal with $10 \mathrm{~mm}$ edges was used as a specimen. In order to indent the specimen, a line load parallel to the [110] direction of the crystal specimen was applied into the (001) surface of the specimen by a tungsten carbide wedge indenter with an included angle of $90^{\circ}$. The nominal total depth of indentation was $\sim 200 \mu \mathrm{m}$, and after unloading the final indentation depth was measured as $\sim 160 \mu \mathrm{m}$ which is very small compared to the dimensions of the specimen so as to maintain small scale yielding conditions. The force vs. displacement relation was measured during the indentation process as shown in Fig. 2.

\subsection{Effective In-plane Slip Systems}

The crystallographic slip systems in an FCC single crystal are known to be in the [110] directions and on $\{111\}$ close packed planes. The smallest possible perfect dislocation Burgers vector is $\frac{1}{2}[110]$ and its magnitude is $b=a / \sqrt{2}$, where $a$ is the lattice parameter (e.g. Hirth \& Lothe, 1992). The effective in-plane slip systems has been used in this study in order to distinguish the crystallographic slip systems from the slip systems created in (110) plane under a load applied parallel to the [110] direction. The orientations of crystallographic slip systems and effective in-plane slip systems are outlined by Kysar et al. (2010) and Kysar et al. (2005).

Rice (1987) showed that under the application of a line load in [110] directions into the surface of (001)-planes, and under the assumption of having the same critical resolved shear 


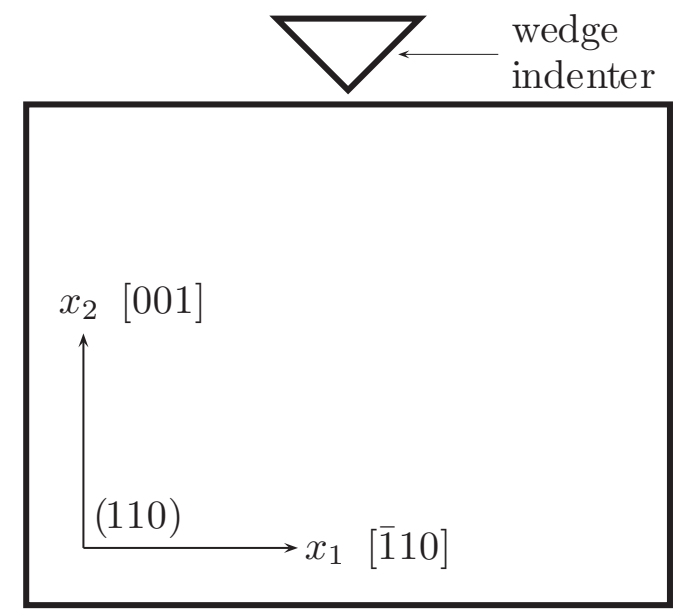

(a)

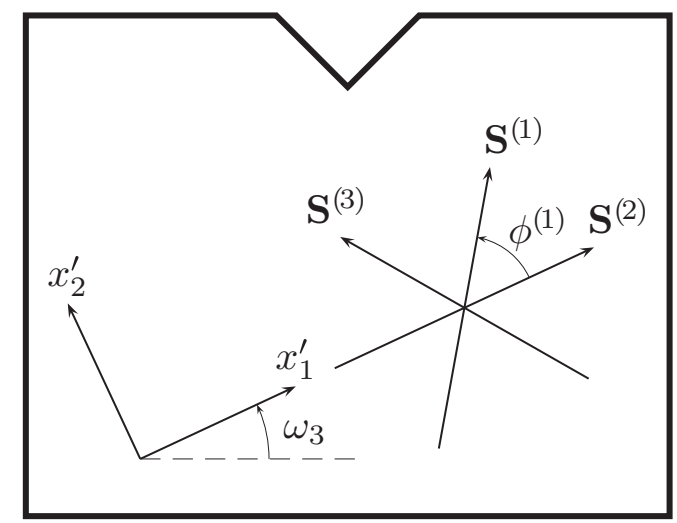

(b)

Figure 3: The single crystal specimen before and after the indentation process: (a) Orientation of nickel single crystal specimen in reference (global) coordinate frame, (b) Orientation of effective slip systems in local crystallographic coordinate frame.

stress $\left(\tau_{C R S S}\right)$, crystallographic slip systems, $(1 \overline{1} 1)[\overline{1} 01]$ and $(1 \overline{1} 1)[011]$, will be activated in equal amounts by experiencing the same resolved shear stress. These coplanar slip systems combine to form an effective slip system on the (111) plane in the direction of [112]. The resulting effective slip system is called Slip System 1, and the unit slip vector of the Slip System 1 is denoted as $\mathbf{S}^{(1)}$, which is oriented at an angle of $\phi_{1}=54.7^{\circ}$. Crystallographic

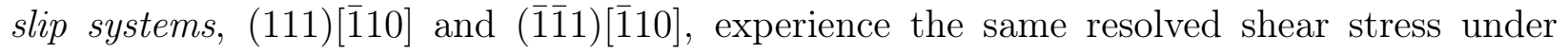
the identical conditions described above. These collinear slip systems combine to form an effective slip system, Slip System 2, on effective (001) planes and [110] slip direction. The unit vector of Slip System 2 is denoted as $\mathbf{S}^{(2)}$ and is oriented at an angle of $\phi_{2}=0^{\circ}$. Similarly,

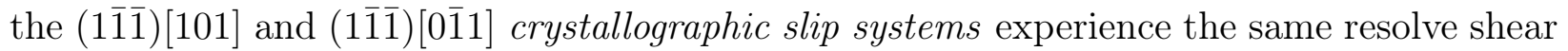
stress, and combine to form the effective in-plane slip system, called Slip System 3. This

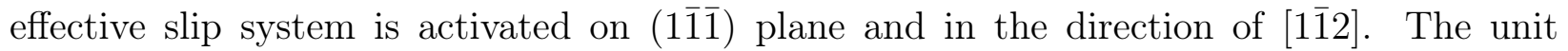
vector of Slip System 3 is denoted as $\mathbf{S}^{(3)}$ and is oriented at an angle of $\phi_{3}=125.3^{\circ}$. The remaining crystallographic slip systems are considered to have no contribution during plane strain plastic deformation (e.g. Kysar et al., 2005 and Kysar et al., 2010).

The orientation of the unit slip vectors, $\mathbf{S}^{(\alpha)}$, depends on $\omega_{3}$ occuring during plastic deformation, as illustrated in Fig. 3. The magnitude of the crystallographic Burgers vector, $b$, is the same for each effective slip system.

\subsection{EBSD Measurements}

After the indentation process, the lattice rotation measurements were performed to capture the lattice rotation field. To do so, a mid-section of the single crystal, which is perpendicular to [110] direction, was exposed with the wire EDM. The newly exposed surface was then electrochemically polished to analyze the plane strain deformation state on this surface using EBSD with a $2.5 \mu \mathrm{m}$ spatial resolution. The rotations about $x_{1}-, x_{2}-$, and 


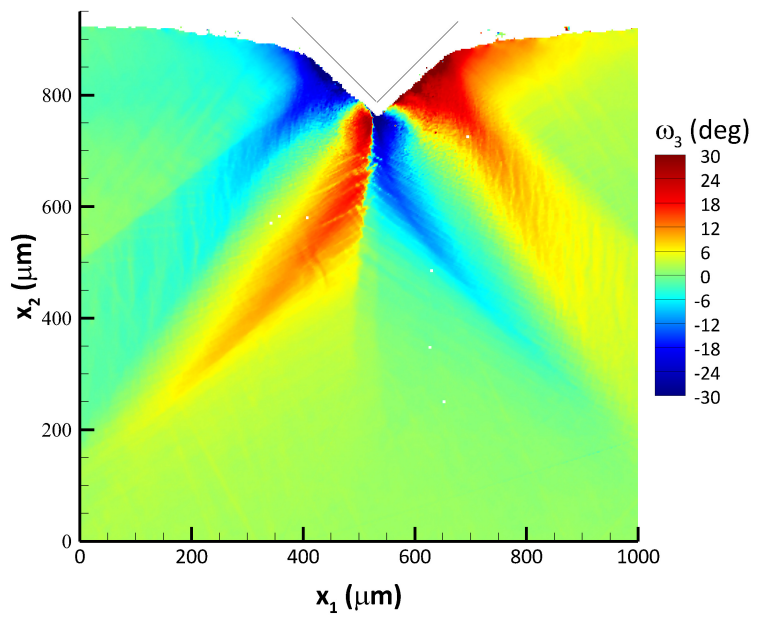

(a)

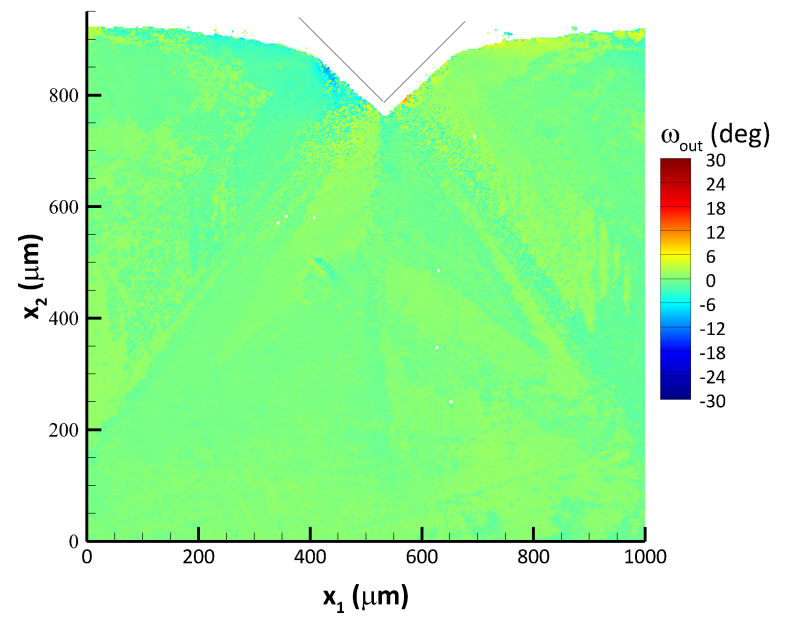

(b)

Figure 4: Experimentally obtained crystal lattice rotations: (a) In-plane lattice rotation, $\omega_{3}$, (rotation about $x_{3}$-axis); (b) Out-of-plane lattice rotation, $\omega_{\text {out }}$.

$x_{3}$-axes are represented by $\omega_{1}, \omega_{2}$, and $\omega_{3}$, respectively. The rotation field of $\omega_{3}$ varies between $-30^{\circ}$ and $+30^{\circ}$ as shown in Fig. $4(\mathrm{a})$. It can also be observed that the rotation field is antisymmetric about the midline, which is the vertical axis passes through the indenter tip, and there is a jump discontinuity on the midline.

In-plane lattice rotation is the counterclockwise rotation about $x_{3}$-axis and the counterclockwise rotation is considered as a positive sense of rotation. $\omega_{1}$ and $\omega_{2}$ were measured as zero, which shows that $\omega_{1}=\omega_{2}=0$ is a very good approximation for the plane strain deformation state. The out-of-plane lattice rotation, $\omega_{\text {out }}$ is defined as the angle between $x_{3}^{\prime}$ (i.e. [110] direction of the local crystallographic coordinate frame) and the unit normal of the newly exposed surface of the specimen. The measured out-of-plane lattice rotation is almost zero everywhere on the surface as shown in Fig. 4(b).

\subsection{Spatially Resolved net Burgers Vector Density ( $\beta$-fields)}

The non-zero components of the lattice curvature tensor, Nye's dislocation density tensor, and the $\beta$-fields were obtained from the measurement of the in-plane lattice rotations. The $\beta$-variables vary between $-180^{\circ}$ and $180^{\circ}$ as shown in Fig. 5. The coordinate frame of the $\beta$-field is divided into six sectors as shown in Fig. 6 and Table 1 by specifying the characteristic angles of effective slip systems (i.e. $-180^{\circ},-125.3^{\circ},-54.7^{\circ}, 0^{\circ}, 54.7^{\circ}, 125.3^{\circ}$ and $180^{\circ}$ ). One can easily analyze the $\beta$-field using Table 1 to monitor the activity of slip systems. Each color relates to the activity region of an effective slip system. The regions away from the indenter tip do not experience extreme plastic deformation under the assumption of plane strain $2 \mathrm{D}$ deformation state. A filtering process was applied to the experimental $\beta$-field to determine the boundaries of the slip activity regions more clearly.

The angular range for the $\beta$-variable is related to the orientations of the effective in- 


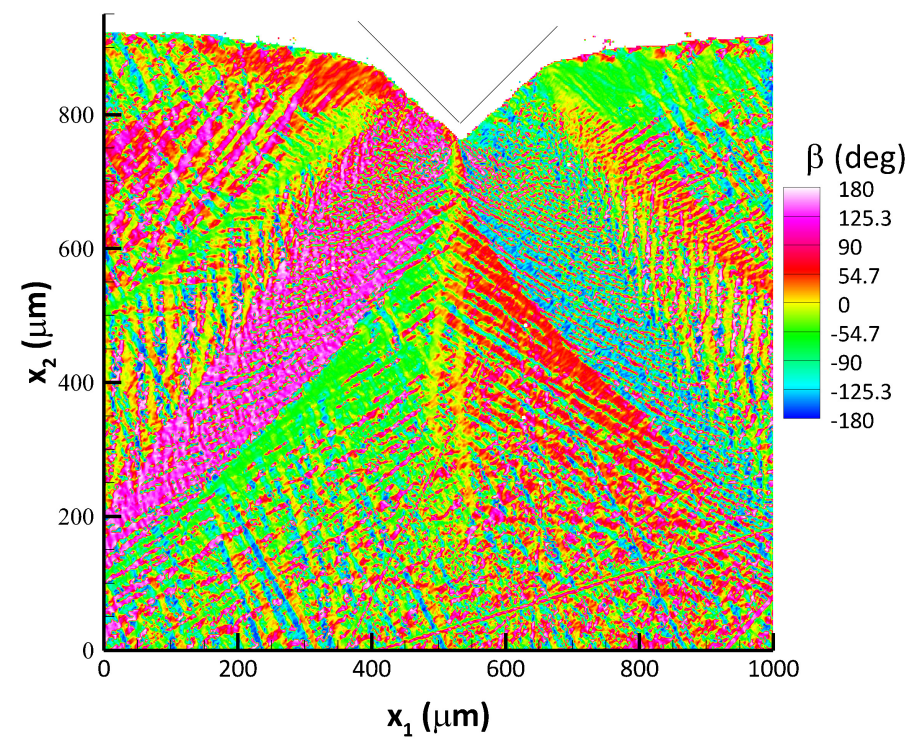

Figure 5: $\beta$-field after the elastic-plastic deformation of the nickel single crystal through a wedge indentation process. Maximum depth of indentation, $a$, is $160 \mu \mathrm{m}$.

plane slip systems as shown in Fig. 6. The range of $\beta$, and the potentially active effective slip systems in those specific ranges are listed in Table 1.

The magnitude of the net Burgers vector density, $B$, was also measured as illustrated in Fig. 7. The maximum value of $B$ was measured as 5 orders of magnitude $\left(m^{-1}\right)$ over the deformed surface. For ease of representation, the data is presented between 3 and 4 orders of magnitude in the contour plot.

\subsection{Filtering of the $\beta$-fields}

In order to eliminate the small variations on the experimental $\beta$-field and to detect the boundaries of the slip activity regions, a filtering process was applied. This enables the comparison of the experimental $\beta$-field to that obtained from the Finite Element Simulations. 2D Low Pass Filtering and 2D Median Filtering were implemented to the experimental $\beta$-field to remove the small variations and striations. Various types of filtering parameters were adjusted using MATLAB commercial software so as to obtain continuous experimental $\beta$-field that can be used as a benchmark against Finite Element Simulation results. To find the suitable filtering parameters, 2D Fast Fourier Transform (2D FFT) was utilized as illustrated in Fig. 8(c) and (d). Less scattering observed in the 2D FFT analysis is an indicator of the less scattering of the small variations in the filtered $\beta$-field. The suitable filtering parameters can also be determined by observing the sharpness of the boundaries of the slip system activity regions. 2D Median Filtering method gave better results compared to the 2D Low Pass Filtering method. 2D Median Filtering creates mxn matrices in two dimensions in the neighborhood of the raw data points. The new output data points generate the median 


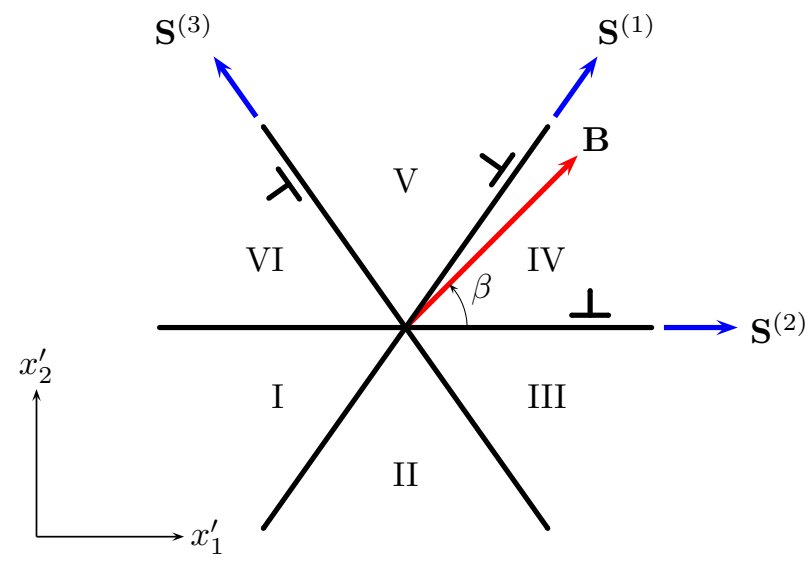

Figure 6: Orientation of the effective in-plane slip systems and the net Burgers vector density in local crystallographic coordinate frame. The coordinate frame is divided into six sectors. Each sector represents a specific range of the $\beta$-variable.

\begin{tabular}{|c|c|c|}
\hline Sector & Range of $\beta$ & $\begin{array}{c}\text { Potentially active } \\
\text { effective slip systems }\end{array}$ \\
\hline \hline I & $-180^{\circ}<\beta<-125.3^{\circ}$ & $-1,-2$ \\
\hline & $\beta=-125.3^{\circ}$ & -1 \\
\hline II & $-125.3^{\circ}<\beta<-54.7^{\circ}$ & $-1,-3$ \\
\hline & $\beta=-54.7^{\circ}$ & -3 \\
\hline III & $-54.7^{\circ}<\beta<0^{\circ}$ & $-2,-3$ \\
\hline & $\beta=0^{\circ}$ & 2 \\
\hline IV & $0^{\circ}<\beta<54.7^{\circ}$ & 1,2 \\
\hline & $\beta=54.7^{\circ}$ & 1 \\
\hline V & $54.7^{\circ}<\beta<125.3^{\circ}$ & 1,3 \\
\hline & $\beta=125.3^{\circ}$ & 3 \\
\hline VI & $125.3^{\circ}<\beta<180^{\circ}$ & 2,3 \\
\hline & $\beta=180^{\circ}$ & -2 \\
\hline
\end{tabular}

Table 1: The range of $\beta$, and the potentially active effective slip systems in that specific range. It is possible to interpret the $\beta$-fields using this table. If $\beta$ equals to the characteristic slip orientation angles such as $-125.3^{\circ},-54.7^{\circ}, 0^{\circ}, 54.7^{\circ}, 125.3^{\circ}$ and $180^{\circ}$, then a single slip system is expected to be activated; if $\beta$ is in a sector, then two slip systems are expected to be activated. 


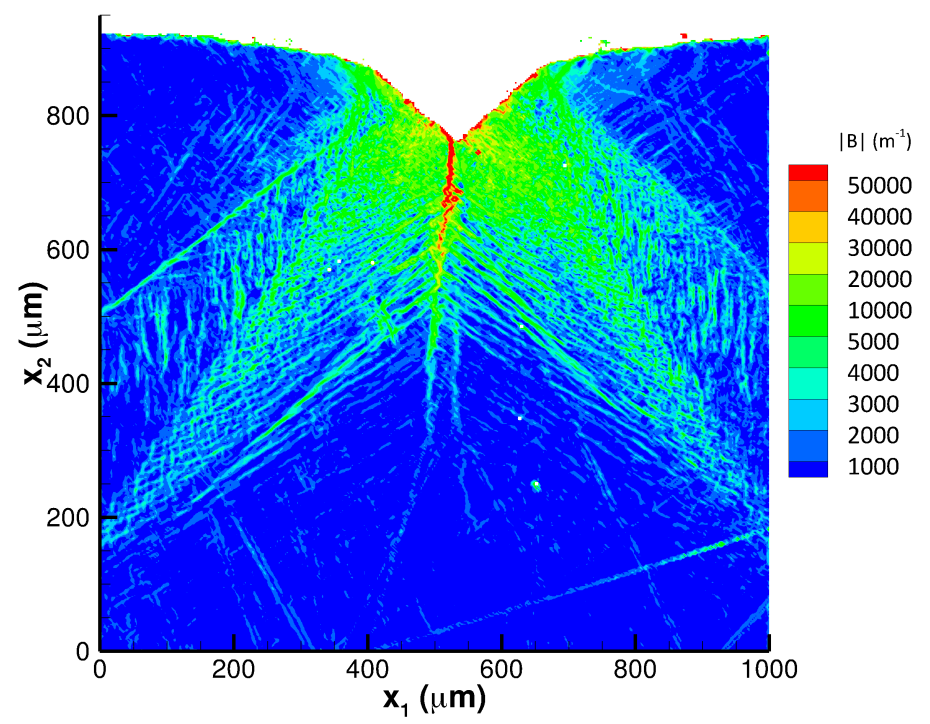

Figure 7: The magnitude of the net Burgers vector density, $B$, after the elastic-plastic deformation of the nickel single crystal.

values in these mxn matrices. The field of new output data points forms a $2 \mathrm{D}$ filtered experimental $\beta$-field as shown in Fig. 8b. 2D FFT was applied to the filtered and unfiltered data as illustrated in Fig. 8c and Fig. 8d to investigate the effect of the spatial frequencies in the vertical and horizontal directions. The spatial frequencies have a significant effect in both vertical and horizontal directions as shown in Fig. 8c. Whereas the low spatial frequency variations represent the boundary value problem, high spatial frequency variations represent the discreteness of the material due to the spatially resolved defect densities. After removing the high frequency spatial variations via filtering process, the boundaries of the slip activity regions, which are represented by different colors, can be detected as shown in Fig. 8(b).

\section{Finite Element Simulations}

A series of Finite Element Simulations were performed to analyze the elastic-plastic behavior of a nickel single crystal through a wedge indentation process. The finite element analysis was carried out using a commercial software package (ABAQUS/Standard, v. 6.10) by employing a UMAT, user-material subroutine, for single crystal plasticity written by Huang (1991) and modified by Kysar (1997). There are two main objectives of the Finite Element Simulations. The first objective is to determine the slip system activities and obtain the $\beta$-fields to characterize the spatial distribution of the net Burgers vector density. The second objective is to analyze the influence of the hardening parameters on the $\beta$-fields. 


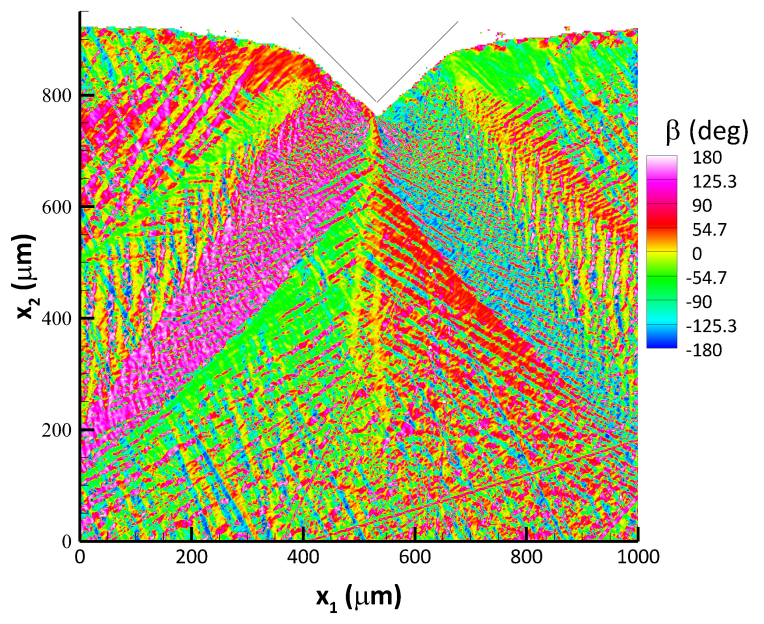

(a)

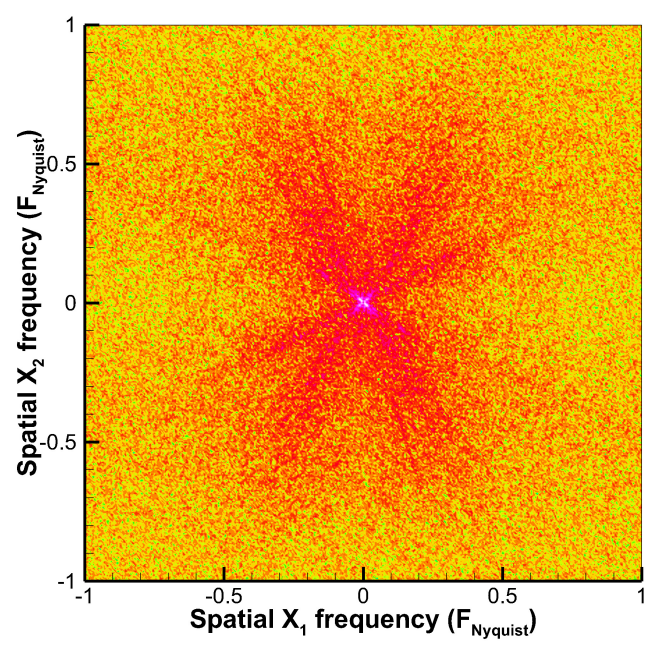

(c)

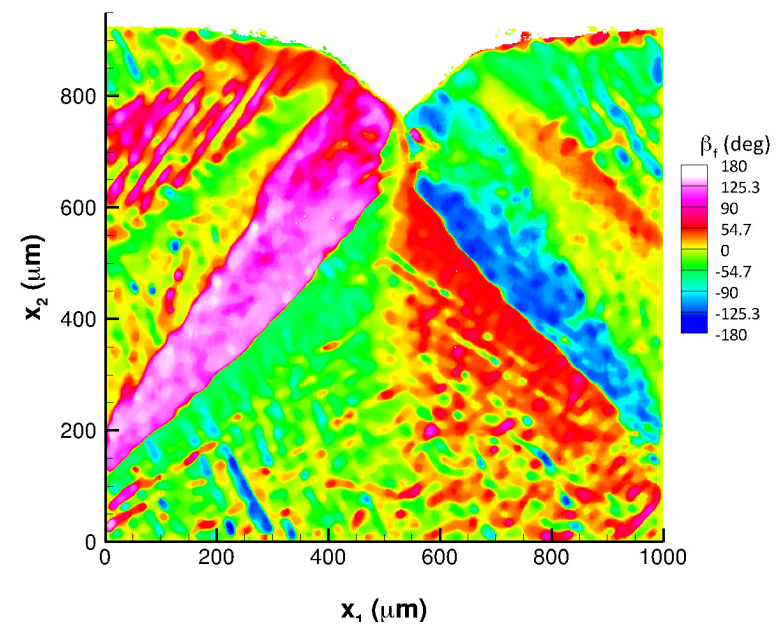

(b)

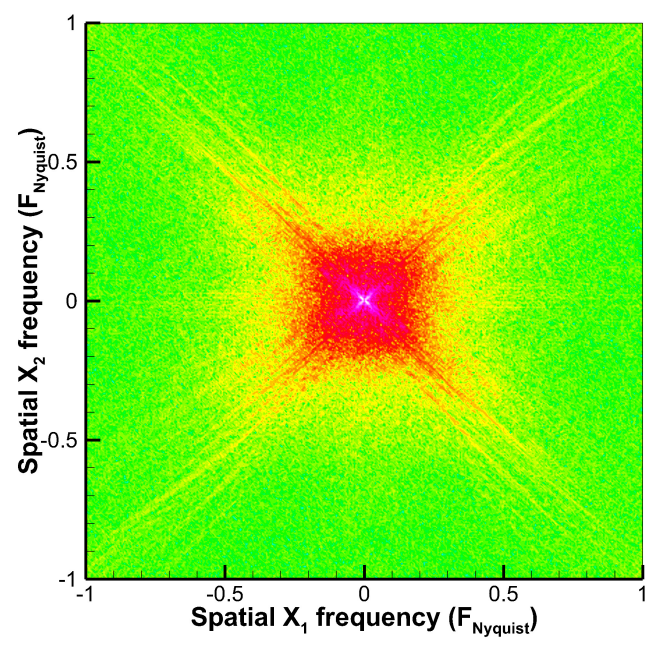

(d)

Figure 8: Two-dimensional (2D) filtering of experimental $\beta$-field: (a) Unfiltered experimental $\beta$-field; (b) 2D filtered experimental $\beta$-field; (c) 2D Fourier Transform of unfiltered $\beta$-field; (d) 2D Fourier Transform of filtered $\beta$-field. 


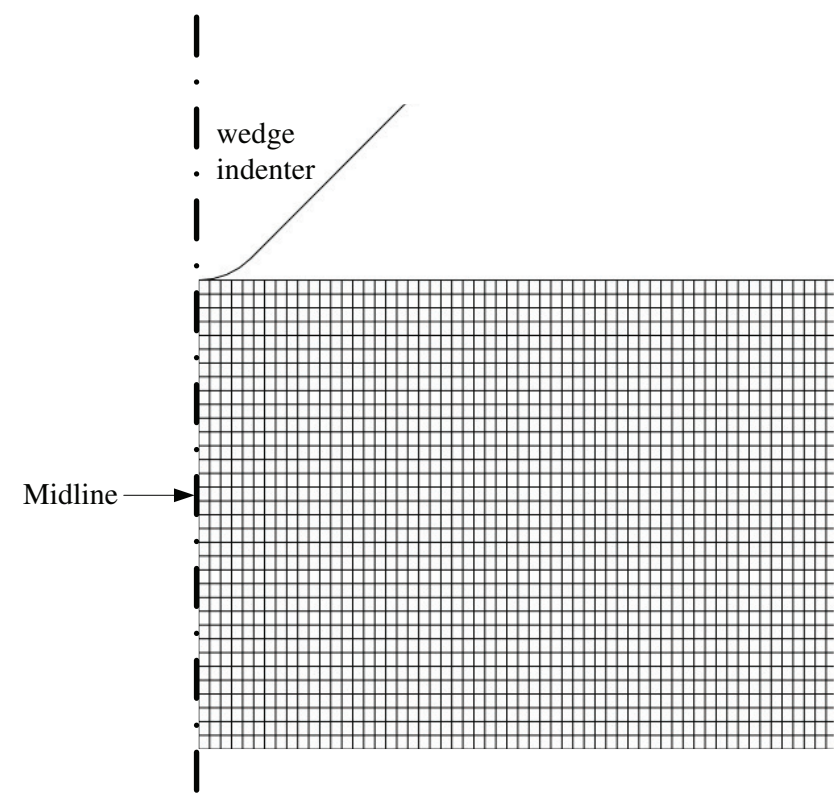

(a)

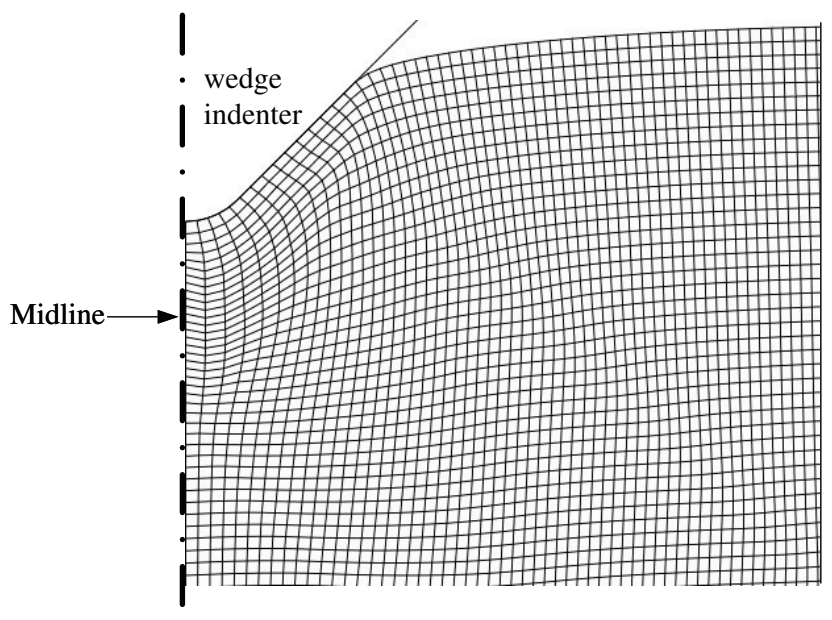

(b)

Figure 9: Finite element mesh: (a) geometry of mesh and rigid indenter; (b) deformed mesh after the indentation process. Note that the figure does not illustrate the whole material mesh. It represents the very top portion of the finer mesh, where extreme plastic deformation takes place.

\subsection{Finite Element Model}

Only the portion of the specimen to the right of the midline was modeled using plane strain bi-linear four-noded quadrilateral elements. A finite element mesh that is suitable for the representation of deformation before and after the indentation process was designed as illustrated in Fig. 9(a) and (b). A 90 -angle wedge indenter with a $100 \mu \mathrm{m}$ radius of curvature at the tip was modeled as a line segment. The deformation that takes place on a small portion of the deformed mesh immediately under the wedge indenter as shown in Fig. 9(b) was analyzed for the simulations. Nodal displacements in the $x_{2}$-direction were constrained to be zero at the base of the specimen, and the nodal displacements in the $x_{1}-$ direction were constrained to be zero on the midline.

Anisotropic elastic properties for nickel (i.e. $C_{11}=246.5 \mathrm{GPa}, C_{12}=147.3 \mathrm{GPa}$, and the $C_{44}=124.7 \mathrm{GPa}$ ) were used (Hirth \& Lothe, 1992). Two phenomenological hardening models with the hardening parameters listed in Table 2 and Table 3 were employed in the Finite Element Simulations. The indenter was modeled as a line segment and displaced quasistatically into the meshed region. A contact algorithm was utilized by ABAQUS to model the contact between the wedge indenter and the single crystal material. The higher friction coefficient between the indenter and material surface gives better results for the distorted mesh immediately under the indenter tip. Thence, the coefficient of friction between the indenter and the material surface was set to 1 by applying small-sliding approximation. In other words, the end point of the indenter tip contacting to the surface does not slide more than an element dimension, and move together with the material while the load is being 


\begin{tabular}{|c|c|c|c|}
\hline Simulation $\#$ & $q$ & $\tau_{s} / \tau_{0}$ & $h_{0} / \tau_{0}$ \\
\hline \hline 1 & 1.0 & 5 & 10 \\
\hline 2 & 1.0 & 5 & 1 \\
\hline 3 & 1.0 & 2 & 10 \\
\hline 4 & 1.0 & 2 & 1 \\
\hline 5 & 1.2 & 5 & 10 \\
\hline 6 & 1.4 & 5 & 10 \\
\hline 7 & 1.6 & 5 & 10 \\
\hline
\end{tabular}

Table 2: The hardening parameters used in the Finite Element Simulations in which material is assumed to show the PAN hardening behavior. The latent hardening ratio $(q)$ varies between 1 and 1.6. The saturation stress $\left(\tau_{s}\right)$ and the initial hardening modulus $\left(h_{0}\right)$ are given in terms of the initial yield stress $\left(\tau_{0}\right)$, which is taken as $1 \mathrm{MPa}$ for each simulation.

\begin{tabular}{|c|c|c|c|c|c|c|}
\hline Simulation $\#$ & $q$ & $\tau_{s} / \tau_{0}$ & $h_{0} / \tau_{0}$ & $h_{s} / \tau_{0}$ & $\gamma_{0}$ & $f_{0}$ \\
\hline \hline 1 & 0 & 2 & 2 & 1 & 0.001 & 7.2 \\
\hline 2 & 0 & 5 & 11 & 1 & 0.001 & 7.2 \\
\hline 3 & 0 & 2 & 11 & 1 & 0.001 & 7.2 \\
\hline 4 & 0 & 5 & 2 & 1 & 0.001 & 7.2 \\
\hline 5 & 0 & 1.24 & 0.7 & 38.7 & 0.001 & 7.2 \\
\hline 6 & 0 & 1.3 & 1.5 & 90 & 0.001 & 8 \\
\hline
\end{tabular}

Table 3: The hardening parameters used in Finite Element Simulations in which material is assumed to show the BW hardening behavior. The latent hardening ratio $(q)$ is taken as 0 for each simulation. The hardening parameters used in simulations $\# 5$ and \#6 are recommended by Kysar (2001) and Bassani \& Wu (1991), respectively.

applied quasistatically.

\subsection{Single Crystal Plasticity}

Hill (1966) showed that the hardening rate of each slip system is a function of the slip rate on all systems. In other words, the hardening rate, $\dot{\tau}$, on each slip system $\alpha$ is described by

$$
\dot{\tau}^{(\alpha)}=\sum_{\beta=1}^{N} h_{\alpha \beta} \dot{\gamma}^{(\beta)},
$$

where $N$ is the total number of slip systems, $\dot{\gamma}$ is the slip rate and $h_{\alpha \beta}$ is the hardening moduli matrix which is determined by different hardening models. The first phenomenological hardening model used in the present work was introduced by Peirce, Asaro, and Needleman (1983), which will be referred to as the PAN hardening model. The second one was introduced by Bassani \& Wu (1991), which will be referred to as the BW hardening model.

The PAN model proposes two hardening moduli, 


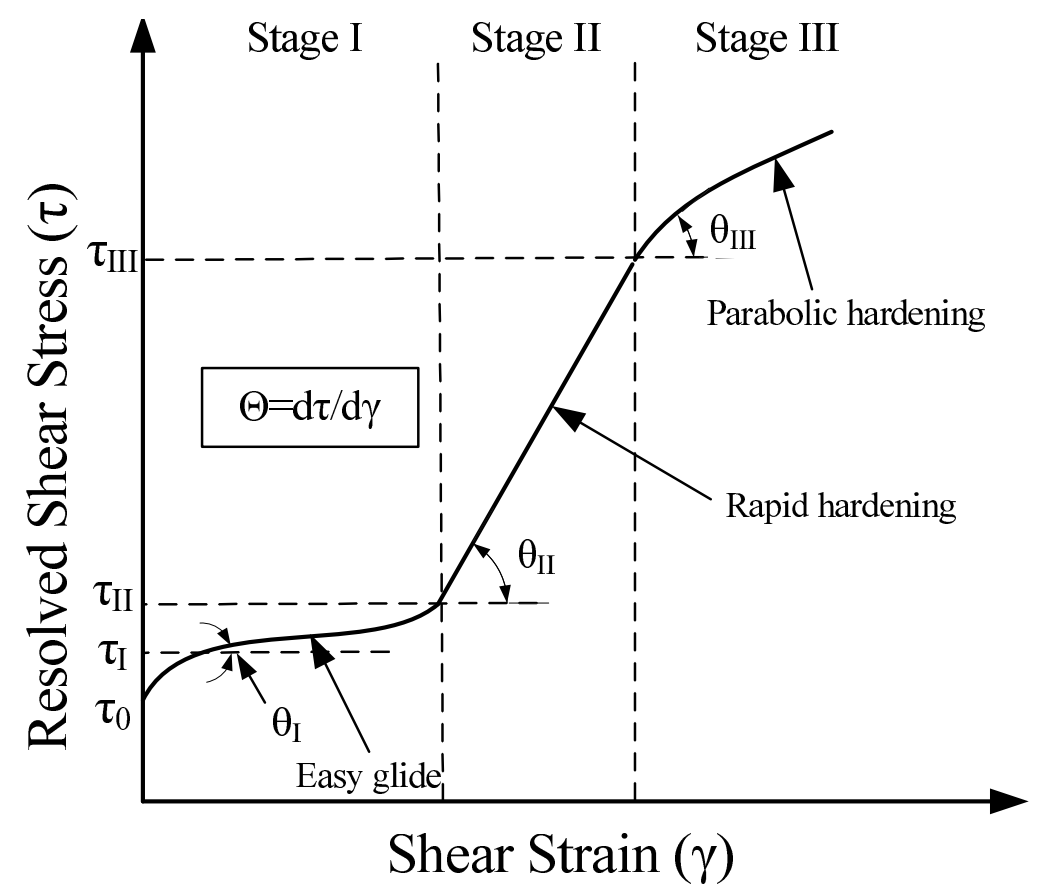

Figure 10: Hardening stages in an FCC single crystal based upon Wu et al. (1991): Easy glide, Rapid hardening and Parabolic hardening

$$
\begin{aligned}
& h_{\alpha \alpha}=h(\gamma)=h_{0} \operatorname{sech}^{2}\left|\frac{h_{0} \gamma}{\tau_{s}-\tau_{0}}\right|, \\
& h_{\alpha \beta}=q h(\gamma) \quad(\alpha \neq \beta),
\end{aligned}
$$

where $h_{\alpha \alpha}$ term is known as the self-hardening modulus, $h_{0}$ is the initial hardening modulus, $\tau_{s}$ is the saturation strength, $\tau_{0}$ is the initial yield stress (i.e. $\tau_{0}=\tau^{(\alpha)}(0)$ ). The term $h_{\alpha \beta}$ (for $\alpha \neq \beta$ ) is known as the latent hardening modulus, and $q$ is the latent hardening ratio, which is the ratio of the latent hardening rate to the self hardening rate of a slip system. The total accumulated strain on all slip systems, the total slip, is given by

$$
\gamma=\sum_{\alpha=1}^{N} \int_{0}^{t}\left|\dot{\gamma}^{(\alpha)}\right| d t .
$$

Bassani \& Wu (1991) proposed a hardening model, which decribes the three hardening stages of an FCC single crystal as shown in Fig. 10. The self hardening (active hardening) and latent hardening moduli are expressed by the BW model as 


$$
\begin{aligned}
& h_{\alpha \alpha}=F\left(\gamma^{(\alpha)}\right) G\left(\gamma^{(\beta)} ; \beta=1, N, \beta \neq \alpha\right), \\
& F\left(\gamma^{(\alpha)}\right)=\left[\left(h_{0}-h_{s}\right) \operatorname{sech}^{2}\left|\frac{\left(h_{0}-h_{s}\right) \gamma^{(\alpha)}}{\tau_{s}-\tau_{0}}\right|+h_{s}\right], \\
& G\left(\gamma^{(\beta)} ; \beta \neq \alpha\right)=1+\sum_{\beta \neq \alpha} f_{\alpha \beta} \tanh \left(\frac{\gamma^{(\beta)}}{\gamma_{0}}\right), \\
& h_{\alpha \beta}=q h_{\alpha \alpha} \quad(\alpha \neq \beta),
\end{aligned}
$$

where $h_{s}$ is the hardening modulus during easy glide, $F\left(\gamma^{(\alpha)}\right)$ is the instantaneous hardening modulus under single slip, and $G$ is the function taking into account the effect of crosshardening or interaction of slip systems. It can be inferred from the equation above that during the easy glide stage, $G=1$ and the slip hardening behavior is represented by the function $F$. In the rapid hardening stage, due to the interaction between the primary and secondary slip, the forest hardening occurs and this hardening behavior is represented by the function $G . \gamma_{0}$ is the strain level after which the interaction between slip system $\alpha$ and slip system $\beta$ reaches the peak strength. $f_{\alpha \beta}$ denotes the strength of the slip interaction between the slip systems.

The recommended values of the latent hardening ratio is in the range of $1 \leq q \leq 1.6$ for the PAN hardening model. The special case of Taylor, or isotropic, hardening is given by $q=1.0$. In this case, due to the isotropic hardening, the hexagonal yield locus expands equally in all directions in stress space. If $q>1.0$, then latent slip systems harden faster than the active slip systems (Kocks, 1970). The facets of the yield locus correspond to a single slip and the vertices correspond to a double slip. $q=0$ is referred to as diagonal hardening, and it is the recommended value for the BW model. Diagonal hardening effect can be observed either at vertex or facet of the yield locus. In the case of diagonal hardening observed at a vertex, the three facets of the yield locus extend to form a triple stress point representing triple slip, and the latter is observed as the offset of two facets of the initial yield locus (Cuitino \& Ortiz, 1996).

\section{Comparisons of the Results}

In-plane and out-of-plane lattice rotations were measured using EBSD, and experimental $\beta$-fields were obtained after the deformation of a nickel single crystal by a wedge indenter in order to probe the slip system activity regions. Finite Element Simulations pertaining to two distinct hardening models were performed to capture the boundaries of the slip activity regions defined by each $\beta$-field. To be able to compare the experimentally obtained $\beta$-fields with those obtained from the Finite Element Simulations, a filtering process was applied. In the comparisons, the right half portion of the deformed single crystal experiencing the high plastic deformation immediately under the indenter tip was monitored. A novel approach illustrating the directions of the net Burgers vector density over the deformed surface through the streamtraces is presented for both finite element analysis and experimental results. 


\subsection{Comparison of the Experimental and PAN Simulation Results}

The $\beta$-fields obtained from the Finite Element Simulations, which employ the PAN hardening model, were compared with the $\beta$-field obtained experimentally to determine the correct set of hardening parameters. To do so, the $\beta$-fields were utilized to monitor the activity of slip systems in deformed region. The boundaries of the slip activity regions that are represented by different color scheme were compared for different set of parameters listed in Table 2. Depending upon the simulation parameters, whereas the $\beta$-fields obtained from Simulations $\# 1, \# 5, \# 6$ and $\# 7$ have good agreement with the $\beta$-field obtained from experimental measurements as shown in Fig. 11, the Simulations $\# 2, \# 3$ and $\# 4$ have no agreement with the experimental $\beta$-field as illustrated in Fig. 12. The reason for presenting the figures that do not fit the experimentally obtained data is to show how the $\beta$-fields are sensitive to the hardening parameters.

Simulation \#6 and simulation $\# 7$ have identical $\tau_{s} / \tau_{0}$ and $h_{0} / \tau_{0}$ ratios, which are 5 and 10 , respectively. The latent hardening ratios $(q)$ are slightly different from each other as shown in Fig. 11. The latent hardening ratios selected for the simulations are the recommended values for Nickel Single Crystal as discussed in Section 4.2. The predominant color pattern pertaining to the $\beta$-fields is red, blue, yellow, red and green, successively as shown in Fig. 11(b) and Fig. 11(c). The $\beta$-fields can be interpreted by using the legend associated with the characteristic orientation angles of the slip systems. The red color represents $54.7^{0}$, which is the characteristic orientation angle of Slip System 1. In other words, only the Slip System 1 is activated in the red zone. The blue region is represented by the angle varying between $-125.3^{0}$ and $-180^{\circ}$, that is Slip System -1 and Slip System - 2 are activated together in this region. Similarly, yellow region is represented by $0^{0}$, and this angle indicates that only the Slip System 2 is activated in this region. The Slip System -3 is predominantly active in the green region, and the field close to the (001) surface, which is away from the indentation tip, indicates an elastic deformation to be analyzed later.

In order to obtain the vector representation of the net Burgers vector density, a streamtrace analysis was performed by employing Tecplot 360 commercial software. Experimentally measured $\alpha_{13}^{\prime}$ and $\alpha_{23}^{\prime}$, which are $x_{1}^{\prime}$ and $x_{2}^{\prime}$ components of $\mathbf{B}$, were recalculated by applying a filtering process. The combination of the filtered $\alpha_{13}^{\prime}$ and $\alpha_{23}^{\prime}$ components forms the net Burgers vector density as shown in Fig. 13(a). The streamtraces represent the directions of the net Burgers vector density on the deformed surface. For the Finite Element Simulations associated with PAN Hardening Model, the components, $\alpha_{13}^{\prime}$ and $\alpha_{23}^{\prime}$, were directly calculated employing the rotation field obtained through the Finite Element Simulations. The directions of the net Burgers vector density on the deformed surface were compared for experimental and PAN simulation results as illustrated in Fig. 13.

\subsection{Analysis of the $\beta$-fields Along $\operatorname{Arcs}(\beta$ vs. $\theta)$}

To be able to analyze the state of the highly deformed region immediately under the indenter tip, a finer analysis was performed such that the filtered experimental results were compared with the simulation results along the ninety-degree arclengths (i.e. $C_{1}, C_{2}, C_{3}$, and $C_{4}$ ) as shown in Fig. 14 to capture the spatial variations in the $\beta$-variable. This analysis is referred to as $\beta$ vs. $\theta$ analysis in which $\theta$ is the angle in the range of $\left[-90^{0} 0^{0}\right]$, 


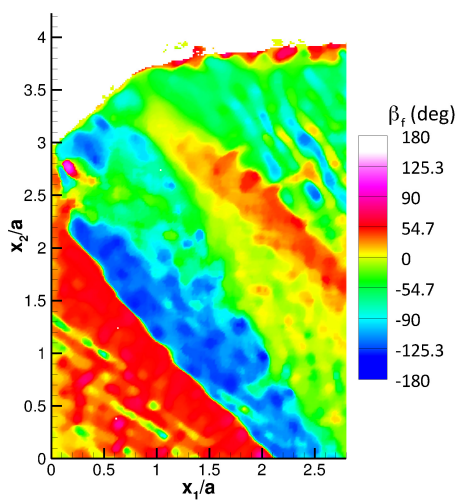

(a)

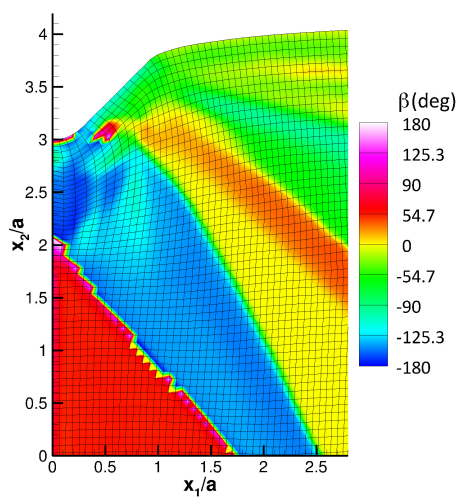

(c)

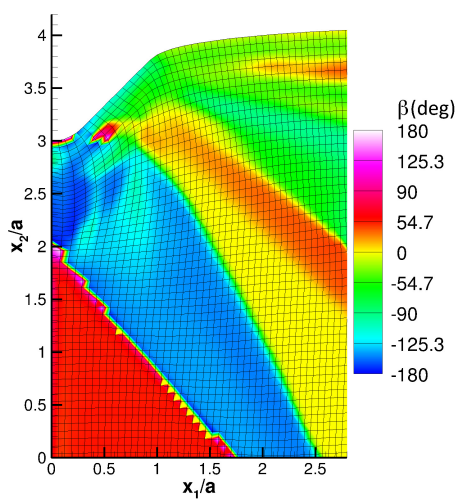

(b)

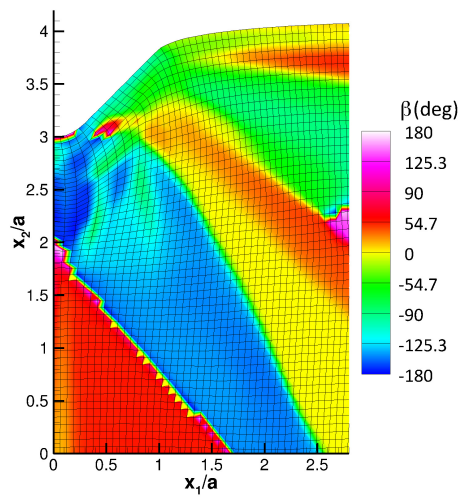

(d)

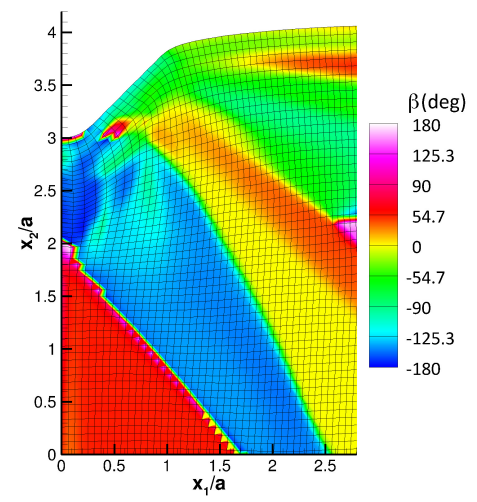

(e)

Figure 11: Comparison of the experimental $\beta$-field to the $\beta$-field associated with the Finite Element Simulations employing PAN Hardening Model: (a) Right half portion of experimental $\beta$-field. The axes are normalized by $a$, maximum depth of indentation; (b) PAN Simulation $\# 6$, parameters: $q=1.4, \tau_{s} / \tau_{0}=5, h_{0} / \tau_{0}=10$; (c) PAN Simulation $\# 7$, parameters: $q=1.6, \tau_{s} / \tau_{0}=5, h_{0} / \tau_{0}=10 ;$ (d) PAN Simulation $\# 1$, parameters: $q=1.0, \tau_{s} / \tau_{0}=5$, $h_{0} / \tau_{0}=10$; (e) PAN Simulation $\# 5$, parameters: $q=1.2, \tau_{s} / \tau_{0}=5, h_{0} / \tau_{0}=10$. 


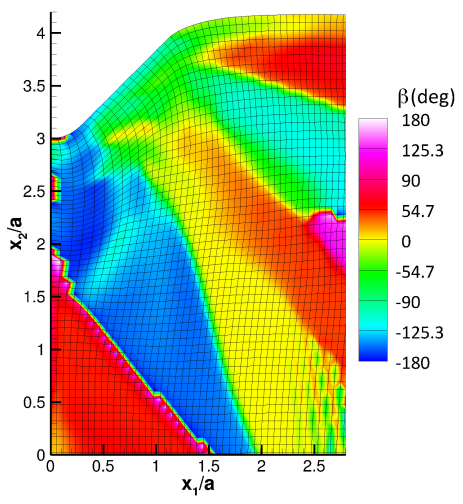

(a)

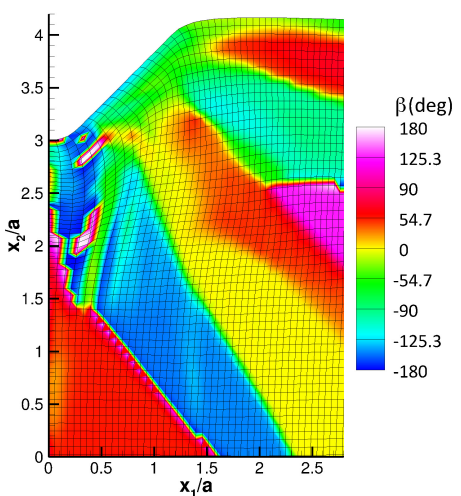

(b)

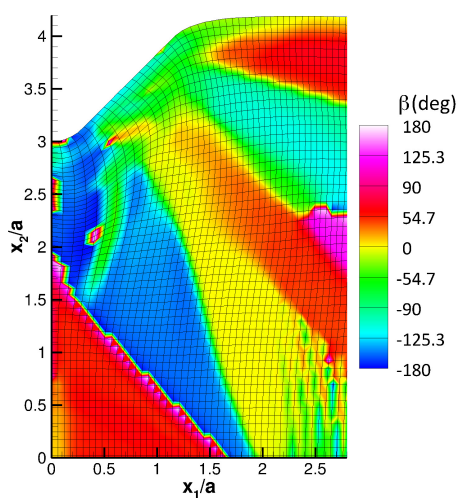

(c)

Figure 12: $\beta$-fields obtained from PAN Finite Element Simulations that do not fit the experimental $\beta$-field: (a) PAN Simulation $\# 2$, parameters: $q=1.0, \tau_{s} / \tau_{0}=5, h_{0} / \tau_{0}=1$; (b) PAN Simulation $\# 3$, parameters: $q=1.0, \tau_{s} / \tau_{0}=2, h_{0} / \tau_{0}=10$; (c) PAN Simulation $\# 4$, parameters: $q=1.0, \tau_{s} / \tau_{0}=2, h_{0} / \tau_{0}=1$. 


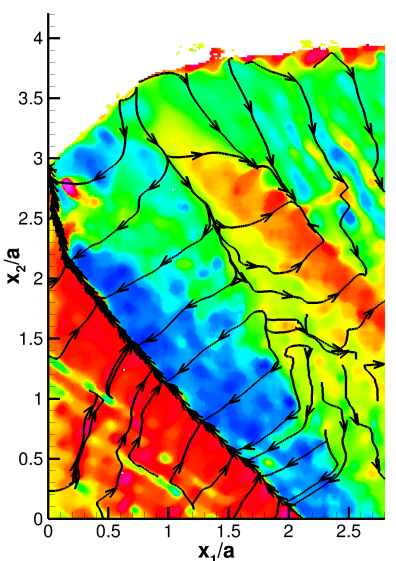

(a)

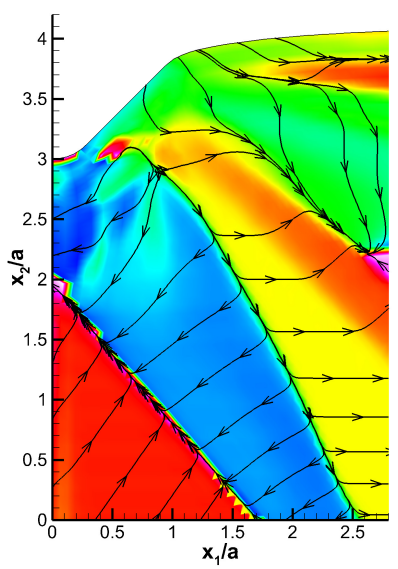

(c)

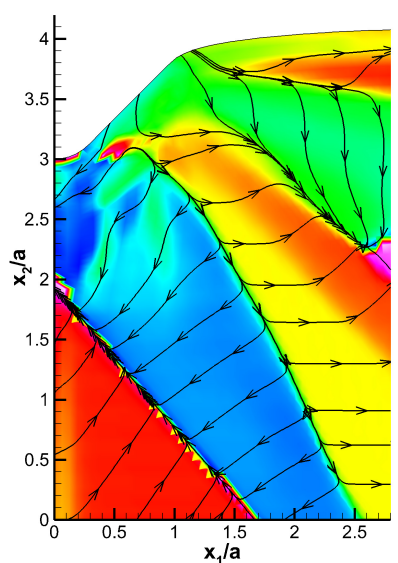

(b)

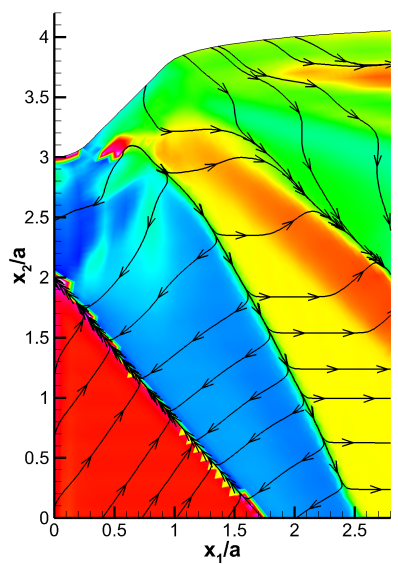

(d)

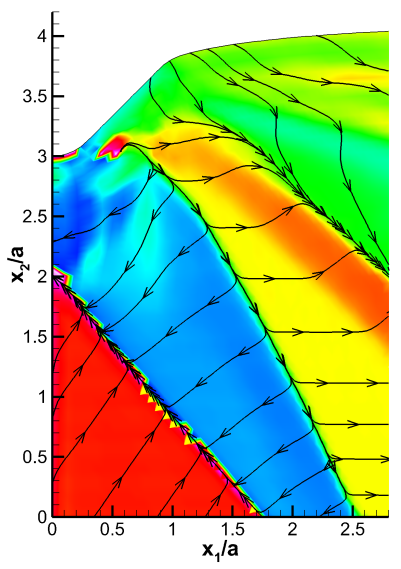

(e)

Figure 13: $\beta$-fields with the directions of the net Burgers vector density: (a) experimental $\beta$-field; (b) PAN Simulation \#1; (c) PAN Simulation \#5; (d) Simulation \#6; (d) PAN Simulation $\# 7$. 


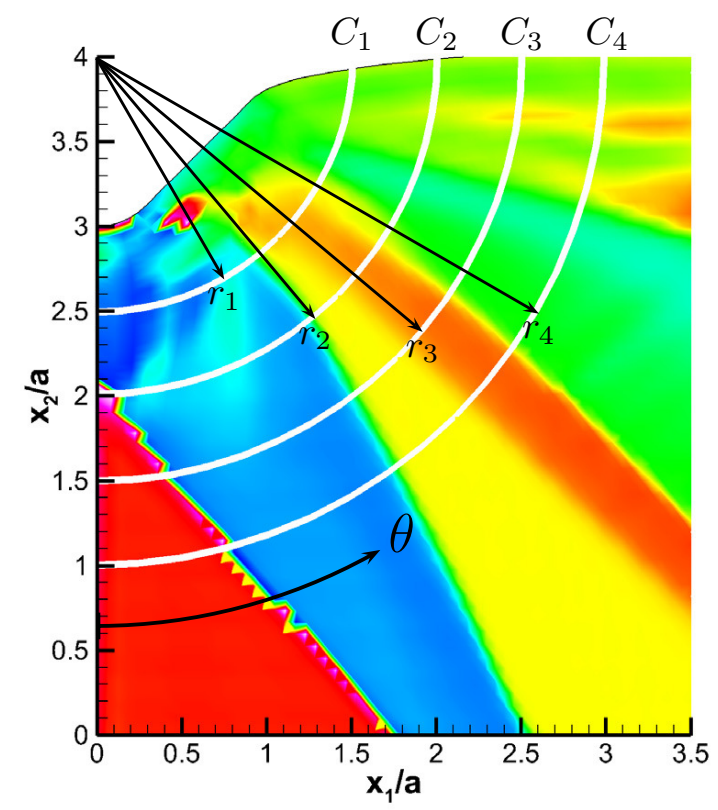

Figure 14: Construction of $\beta$ vs. $\theta$ Analysis: $r_{1}=1.5 a, r_{2}=2 a, r_{3}=2.5 a$, and $r_{4}=3 a$.

and the axes are normalized by $a$. The radii of the 90-degree arcs that are used for the analysis are $r_{1}=1.5 a, r_{2}=2 a, r_{3}=2.5 a$, and $r_{4}=3 a$, respectively. As a sample case, the filtered experimental data was extracted and compared with the data associated with PAN Simulation $\# 1$ as shown in Fig. 15. Whereas the as-filtered $\beta$-values along $C_{1}$ and $C_{2}$, which are close to the tip of the wedge indenter as shown in Fig. 15(a) and Fig. 15(b), do not have a good agreement with the $\beta$-variables obtained from the Finite Element Simulations; the $\beta$-variables along $C_{3}$ and $C_{4}$, have a good agreement with those obtained from the Finite Element Simulations as illustrated in Fig. 15(c) and Fig. 15(d). This can be attributed to the relatively large rotations, which take place in the area between the indenter tip and $C_{2}$.

\subsection{Comparison of the Experimental and BW Simulation Results}

A similar comparative analysis was performed for the Finite Element Simulations associated with the BW Model employing the hardening parameters listed in Table 3. Whereas the $\beta$-fields obtained from Simulations $\# 5$ and $\# 6$ have good agreement with that obtained from experimental measurements as shown in Fig. 16, the Simulations $\# 1, \# 2$, \#3 and \#4 have no agreement with the experimental $\beta$-field as illustrated in Fig. 17 . Diagonal hardening rule was considered for each simulation case, and the results obtained from the simulations having $q=0, \tau_{s} / \tau_{0}=1.24, h_{0} / \tau_{0}=0.7, h_{s} / \tau_{0}=38.7, \gamma_{0}=0.001, f_{0}=7.2$ and $q=0, \tau_{s} / \tau_{0}=1.3, h_{0} / \tau_{0}=1.5, h_{s} / \tau_{0}=90, \gamma_{0}=0.001, f_{0}=7.2$ parameter sets are consistent with the experimental results.

A streamtrace analysis was performed also for the BW simulation results by analogy with the objective outlined for the PAN Finite Element Simulations. Similarly, the representation of the net Burgers vector density on the deformed surface was obtained through 


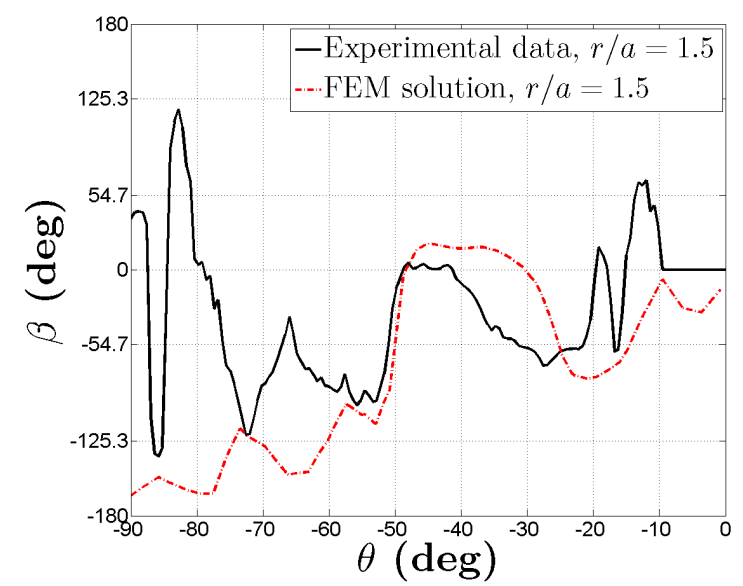

(a)

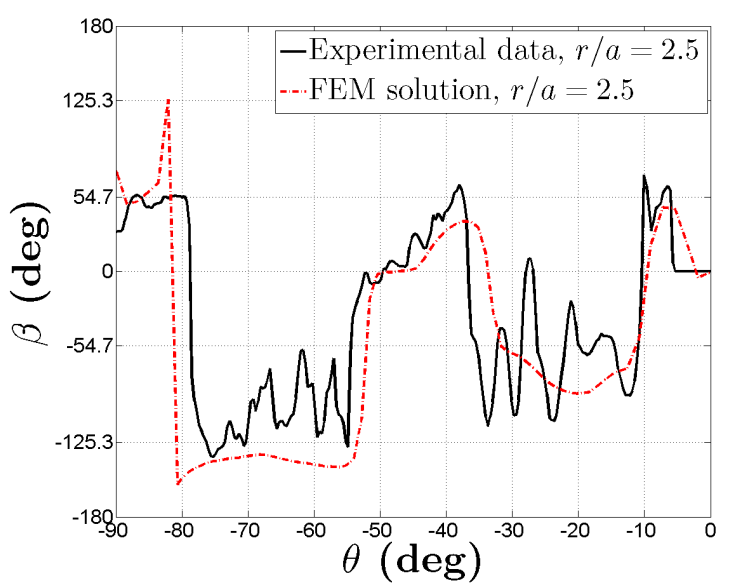

(c)

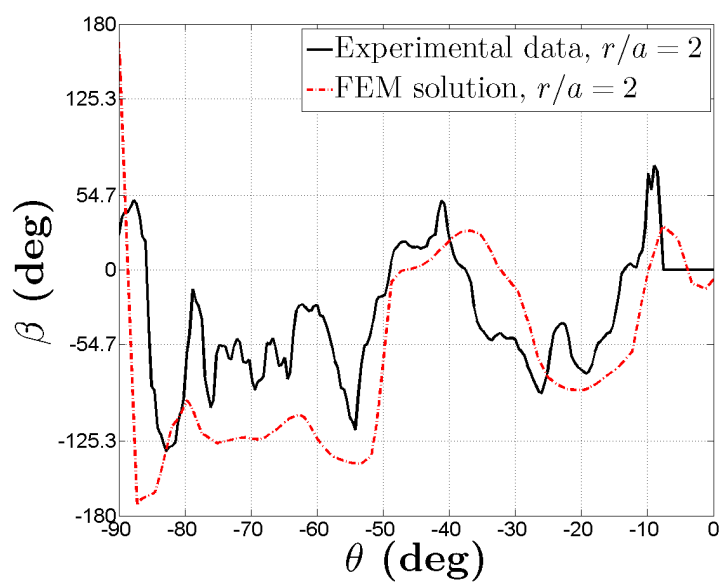

(b)

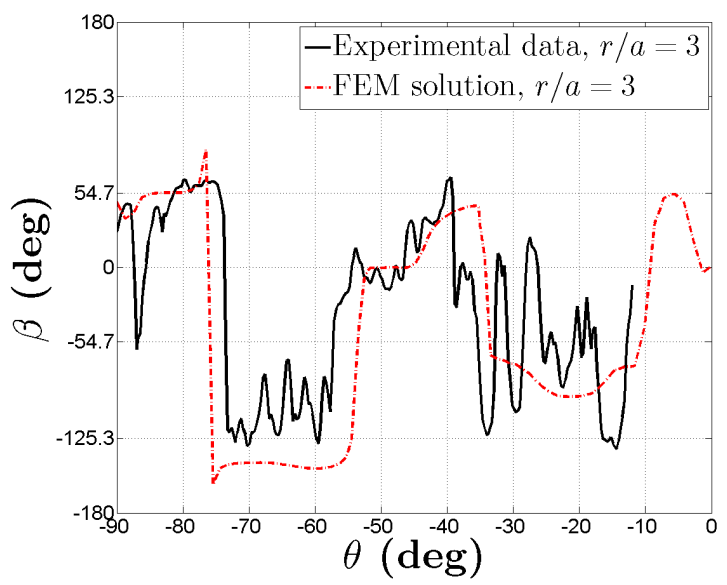

(d)

Figure 15: Variations of the $\beta$ along $C_{1}, C_{2}, C_{3}$, and $C_{4}$ : (a) $\beta$ vs. $\theta$ for $r / a=1.5$; (b) $\beta$ vs. $\theta$ for $r / a=2.0$; (c) $\beta$ vs. $\theta$ curves for $r / a=2.5$; (d) $\beta$ vs. $\theta$ for $r / a=3.0$. 


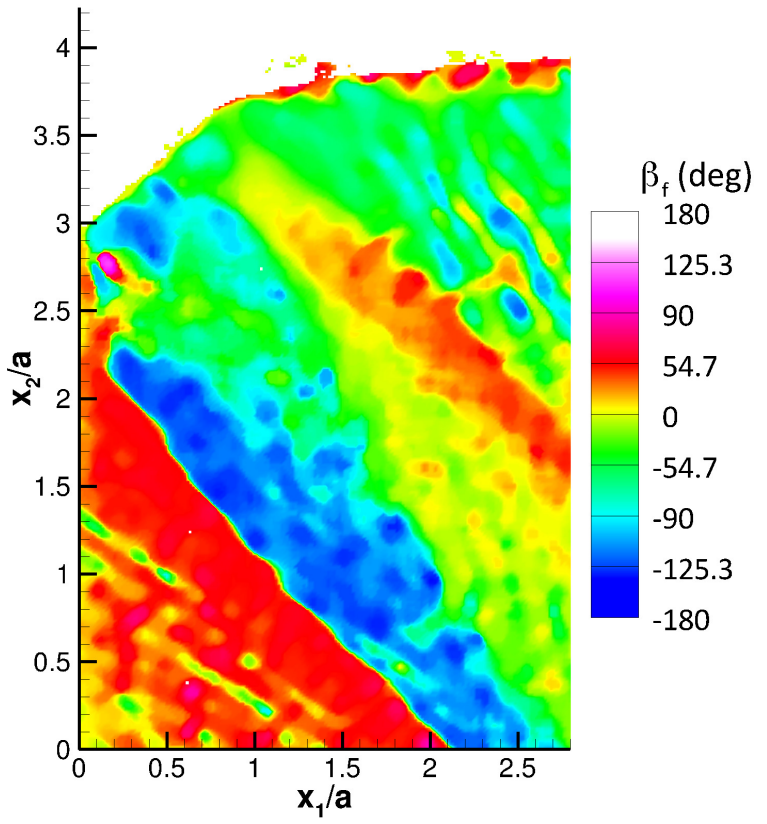

(a)

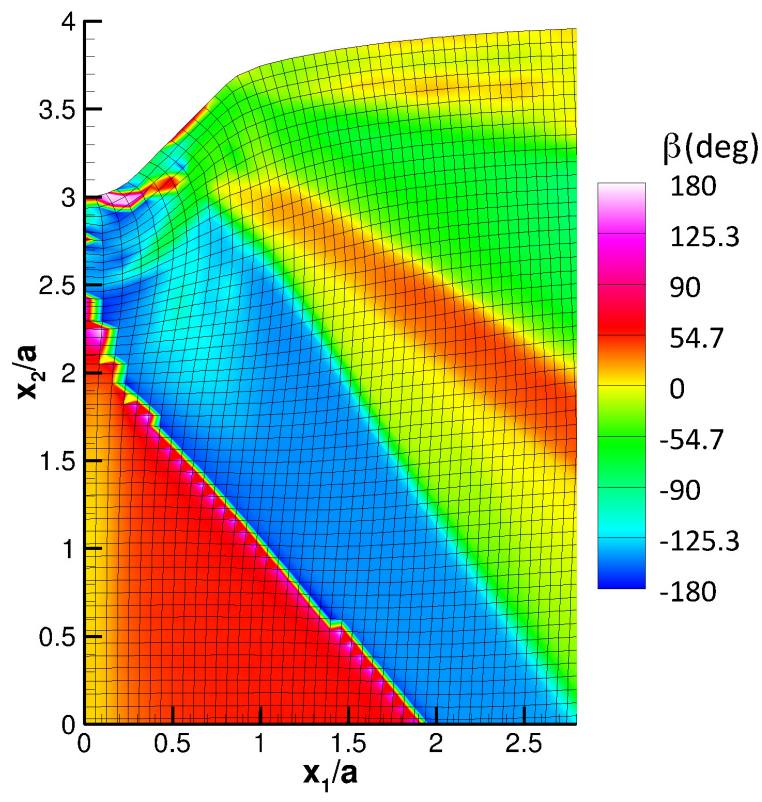

(b)

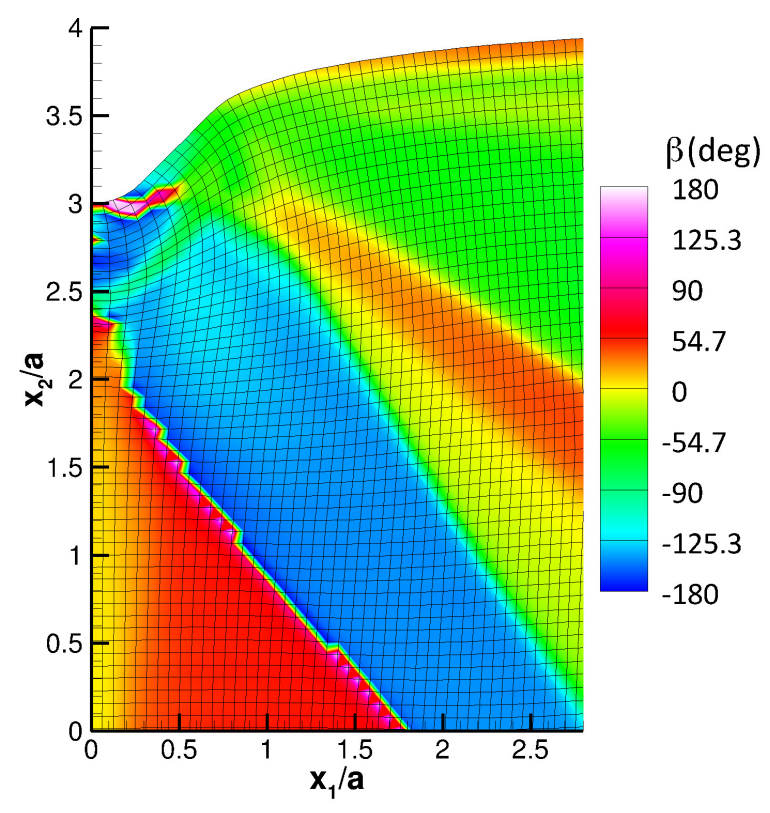

(c)

Figure 16: Comparison of the experimental $\beta$-field and the $\beta$-field associated with the Finite Element Simulations employing BW Hardening Model: (a) Right half portion of the experimental $\beta$-field. The axes are normalized by $a$, maximum depth of indentation; (b) BW Simulation \#5, parameters: $q=0, \tau_{s} / \tau_{0}=1.24, h_{0} / \tau_{0}=0.7, h_{s} / \tau_{0}=38.7, \gamma_{0}=0.001$, $f_{0}=7.2$; (c) BW Simulation \#6, parameters: $q=0, \tau_{s} / \tau_{0}=1.3, h_{0} / \tau_{0}=1.5, h_{s} / \tau_{0}=90$, $\gamma_{0}=0.001, f_{0}=7.2$. 


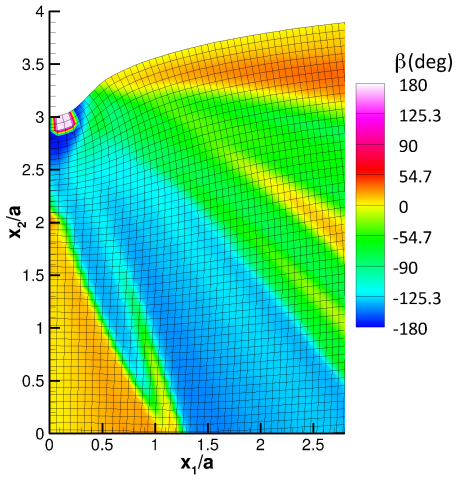

(a)

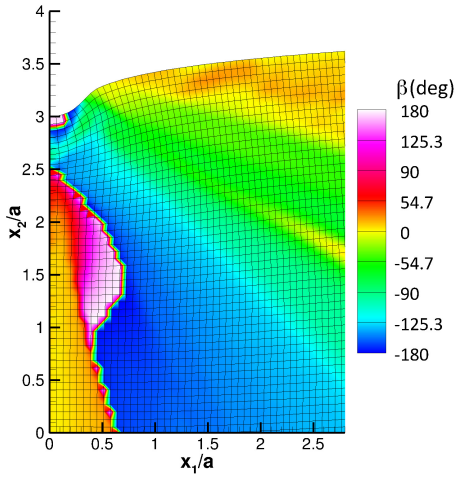

(c)

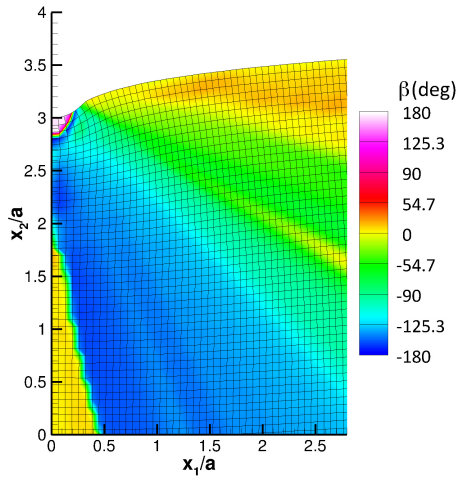

(b)

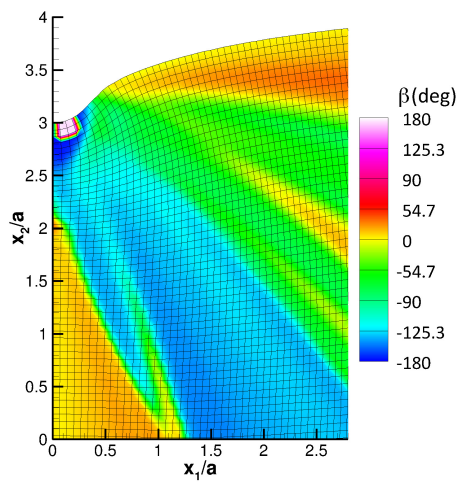

(d)

Figure 17: $\beta$-fields obtained from BW Finite Element Simulations that do not fit the experimental $\beta$-field: (a) BW Simulation \#1, parameters: $q=0, \tau_{s} / \tau_{0}=2, h_{0} / \tau_{0}=2$, $h_{s} / \tau_{0}=1, \gamma_{0}=0.001, f_{0}=7.2$; (b) BW Simulation \#2, parameters: $q=0, \tau_{s} / \tau_{0}=5$, $h_{0} / \tau_{0}=11, h_{s} / \tau_{0}=1, \gamma_{0}=0.001, f_{0}=7.2$; (c) BW Simulation \#3, parameters: $q=0$, $\tau_{s} / \tau_{0}=2, h_{0} / \tau_{0}=11, h_{s} / \tau_{0}=1, \gamma_{0}=0.001, f_{0}=7.2$; (d) BW Simulation \#4, parameters: $q=0, \tau_{s} / \tau_{0}=5, h_{0} / \tau_{0}=2, h_{s} / \tau_{0}=1, \gamma_{0}=0.001, f_{0}=7.2$. 
streamtraces as illustrated in Fig. 18. The directions defined by the streamtraces revealed that the streamtraces follow the boundaries of the slip system activity regions. This gives direct information about the state of the material by quantifying the both direction and the magnitude of the net Burgers vector density.

\section{Conclusions}

Experimental methods currently in use aim to define the elastic-plastic constitutive behavior of a material in terms of stress vs. strain, force vs. displacement, torsion vs. twist and moment vs. curvature relations. These methods are insufficient to completely characterize the deformed state of the material. In this study, a new parameter $(\beta)$ has been introduced to validate the existing constitutive models. The $\beta$-variable is essentially the orientation angle of the net Burgers density vector, obtained as the net signed dislocations per enclosed area.

The analysis presented herein gives direct information about the state of a single crystal undergoing extreme plastic deformation, and also gives a full description of the deformation state of the material in light of the spatially resolved net Burgers vector density (magnitude and direction) fields. In particular, the deformation state of a nickel single crystal has been investigated. The experimental procedure and the single crystal plasticity simulations are detailed in the present study.

The net Burgers vector density and its orientation $(\beta)$ are defined and derived analytically by employing the elastic incompatibility equation and Nye's dislocation density tensor. The relationships between the $\beta$-variable and characteristic slip orientation angles are also presented. Spatially resolved net Burgers vector density fields, $\beta$-fields, have been determined by employing the spatial gradient of the in-plane lattice rotations that were measured in the range of $\left[-30^{0} 30^{\circ}\right]$ using EBSD method. The out-of-plane lattice orientations were measured as zero on the deformed surface.

The $\beta$-fields are essentially the contour maps having a color scheme that corresponds to the characteristic slip angles. The regions that are separated from each other by the colors represent the activity regions of the slip systems. A two dimensional median filtering and Fast Fourier Transform were applied to the experimentally obtained $\beta$-fields to remove the high spatial frequency variations that represent the discreteness of the material.

Single crystal plasticity simulations were performed by using two different hardening models representing the hardening stages of the FCC single crystal during deformation. The hardening relations and parameters were defined, and the $\beta$-fields were determined through the finite element simulations that serve as a benchmark against experimentally obtained $\beta$ fields. The hardening models employed in the finite element simulations are the PAN and BW simulations. PAN Simulation \#6, in which $q=1.4, \tau_{s} / \tau_{0}=5, h_{0} / \tau_{0}=10$, and Simulation $\# 7$, in which $q=1.6, \tau_{s} / \tau_{0}=5, h_{0} / \tau_{0}=10$, give consistent results with experimentally obtained $\beta$-fields. BW Simulation $\# 5$, where $q=0, \tau_{s} / \tau_{0}=1.24, h_{0} / \tau_{0}=0.7, h_{s} / \tau_{0}=38.7$, $\gamma_{0}=0.001, f_{0}=7.2$ and $q=0, \tau_{s} / \tau_{0}=1.3, h_{0} / \tau_{0}=1.5, h_{s} / \tau_{0}=90, \gamma_{0}=0.001, f_{0}=7.2$ give consistent results with experimentally obtained $\beta$-fields. In order to get finer information about the state of the material, an analysis was performed to extract $\beta$-variable along the 90-degree arc-lengths. The directions of the net Burgers density vectors were inserted to the 


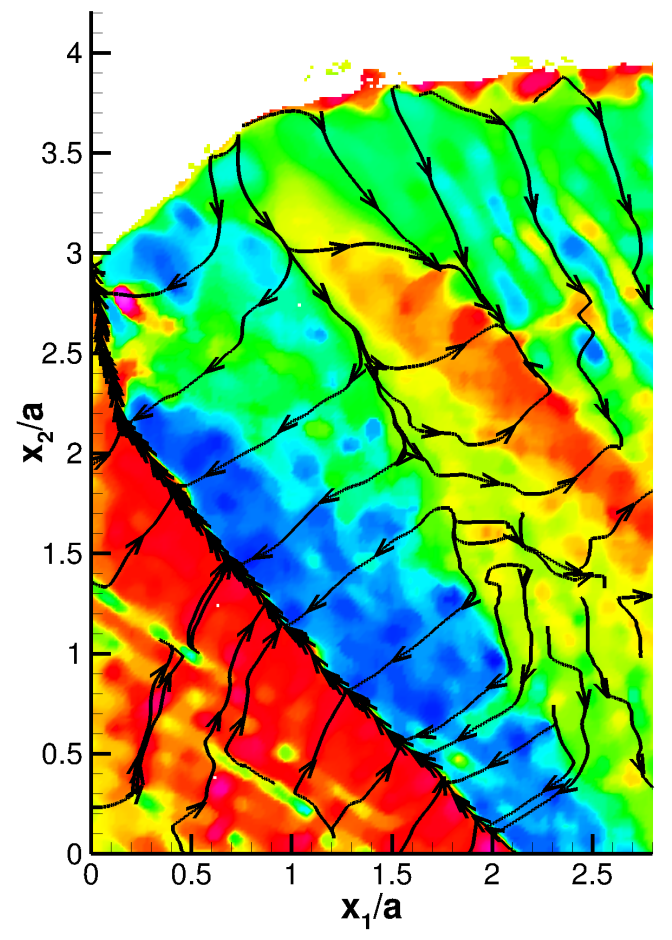

(a)

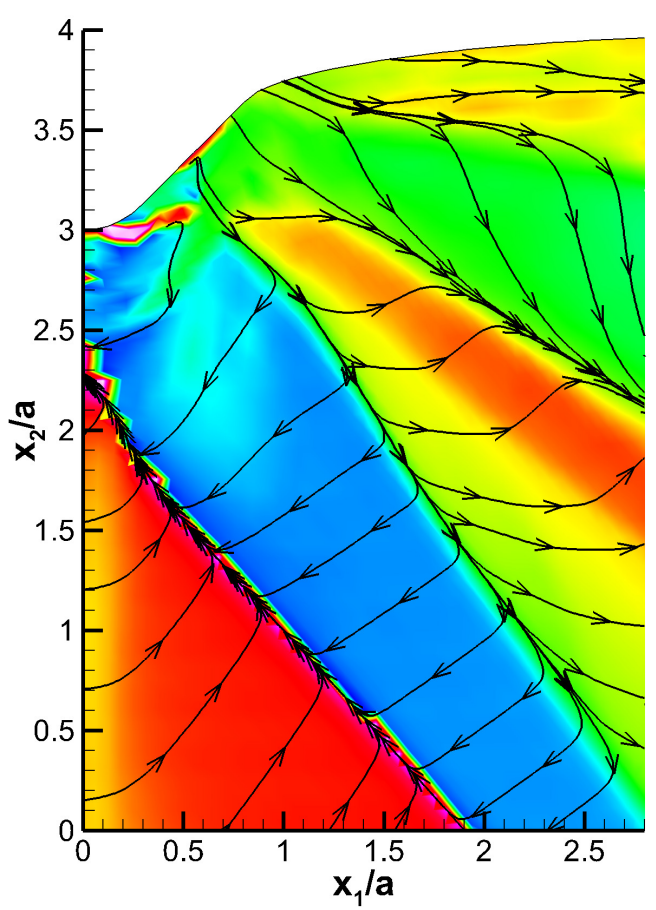

(b)

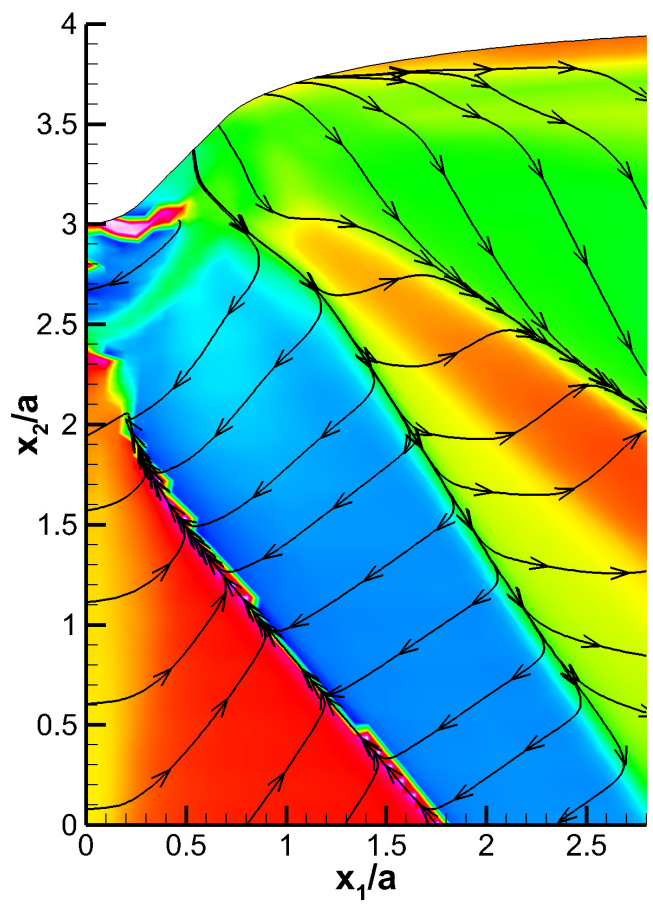

(c)

Figure 18: $\beta$-fields with the directions of the net Burgers vector density: (a) experimental $\beta$-field; (b) BW Simulation \#5; (c) BW Simulation \#6. 
$\beta$-fields for experimental and simulation cases. The effects of hardening parameters on the $\beta$-fields will be investigated further. 


\section{Acknowledgments}

The authors gratefully acknowledge the support from NSF DMR-1310503. In addition, the authors gratefully acknowledge the support from B. Adams and D. Fullwood. One of the authors (A. S.) also gratefully aknowledges the financial support from Ministry of National Education, Turkey. 


\section{References}

Adams, B. L. (1997). Orientation imaging microscopy: Emerging and future applications. Ultramicroscopy, 67(1-4), 11-17.

Adams, B. L., Wright, S. I., \& Kunze, K. (1993). Orientation imaging: The emergence of a new microscopy. Metallurgical Transactions A, 24(4), 819-831.

Arsenlis, A., \& Parks, D. M. (1999). Crystallographic aspects of geometrically-necessary and statistically-stored dislocation density. Acta Materialia, 47(5), 1597-1611.

Ashby, M. F. (1970). Deformation of plastically non-homogeneous materials. Philosophical Magazine, 21(170), 399-404.

Bassani, J. L., \& Wu, T. Y. (1991). Latent hardening in single crystal II: Analytical characterization and predictions. Proceedings of the Royal Society of London Series A, 435(1893), 21-41.

Beausir, B., \& Fressengeas, C. (2013). Disclination densities from ebsd orientation imaging. International Journal of Solids and Structures, 50(1), 137-146.

Bilby, B. A., Gardner, L. R. T., \& Smith, E. (1958). The relation between dislocation density and stress. Acta Metallurgica, 6, 29-33.

Britton, T. B., \& Wilkinson, A. J. (2011). Measurement of residual elastic strain and lattice rotations with high resolution electron backscatter diffraction. Ultramicroscopy, 111(8), $1395-1404$.

Britton, T. B., \& Wilkinson, A. J. (2012). High resolution electron backscatter diffraction measurements of elastic strain variations in the presence of larger lattice rotations. Ultramicroscopy, 114, 82-95.

Cuitino, A. M., \& Ortiz, M. (1996). Three-dimensional crack-tip fields in four-point-bending copper single-crystal specimens. Journal of the Mechanics and Physics of Solids, 44(6), 863-899, 901-904.

Demir, E., Raabe, D., Zaafarani, N., \& Zaefferer, S. (2009). Investigation of the indentation size effect through the measurement of the geometrically necessary dislocations beneath small indents of different depths using EBSD tomography. Acta Materialia, 57(2), 559569.

El-Dasher, B. S., Adams, B. L., \& Rollett, A. D. (2003). Viewpoint: Experimental recovery of geometrically necessary dislocation density in polycrystals. Scripta Materialia, 48(2), $141-145$.

Field, D. P., Magid, K. R., Mastorakos, I. N., Florando, J. N., Lassila, D. H., \& Morris Jr., J. W. (2010). Mesoscale strain measurement in deformed crystals: A comparison of xray microdiffraction with electron backscatter diffraction. Philosophical Magazine, 90(11), 1451-1464. 
Field, D. P., Trivedi, P. B., Wright, S. I., \& Kumar, M. (2005). Analysis of local orientation gradients in deformed single crystals. Ultramicroscopy, 103(1), 33-39.

Gan, Y. X., Kysar, J. W., \& Morse, T. L. (2006). Cylindrical void in a rigid-ideally plastic single crystal II: experiments and simulations. International Journal of Plasticity, 22(1), $39-72$.

Gardner, C. J., Adams, B. L., Basinger, J., \& Fullwood, D. T. (2010). Ebsd-based continuum dislocation microscopy. International Journal of Plasticity, 26(8), 1234-1247.

Hill, R. (1966). Generalized constitutive relations for incremental deformation of metals crystals by multislip. Journal of the Mechanics and Physics of Solids, 14, 95-102.

Hirth, J. P., \& Lothe, J. (1992). Theory of dislocations. Malabar, Florida: Krieger Publishing Company.

Hofmann, F., Abbey, B., Liu, W., Xu, R., Usher, B. F., Balaur, E., \& Liu, Y. (2013). Xray micro-beam characterization of lattice rotations and distortions due to an individual dislocation. Nature Communications, 4(2774).

Huang, Y. (1991). A user-material subroutine incorporating single crystal plasticity in the ABAQUS finite element program: Mech. Report 178 (Tech. Rep.). Cambridge, Massachusetts: Division of Engineering and Applied Sciences, Harvard University.

Ice, G. E., Larson, B. C., Yang, W., Budai, J. D., Tischler, J. Z., Pang, J. W., .. Liu, W. (2005). Polychromatic x-ray microdiffraction studies of mesoscale structure and dynamics. Journal of Synchrotron Radiation, 12, 155-162.

Jiang, J., Britton, T. B., \& Wilkinson, A. J. (2015). The orientation and strain dependence of dislocation structure evolution in monotonically deformed polycrystalline copper. International Journal of Plasticity, 69, 102-117.

Kiener, D., Pippan, R., Motz, C., \& Kreuzer, H. (2006). Microstructural evolution of the deformed volume beneath microindents in tungsten and copper. Acta Materialia, 54 (10), 2801-2811.

Kocks, U. F. (1970). The relation between polycrystal deformation and single-crystal deformation. Metallurgical and Materials Transactions, 1, 1121-1143.

Kröner, E. (1958). Continuum theory of dislocations and self-stresses. Berlin: SpringerVerlag.

Kysar, J. W. (1997). Addendum to "A user-material subroutine incorporating single crystal plasticity in the ABAQUS finite element program: Mech. Report 178" (Tech. Rep.). Cambridge, Massachusetts: Division of Engineering and Applied Sciences, Harvard University.

Kysar, J. W. (2001). Continuum simulations of directional dependence of crack growth along a copper/sapphire bicrystal interface: Part I: Experiments and crystal plasticity background. Journal of the Mechanics and Physics of Solids, 49(5), 1099-1128. 
Kysar, J. W., \& Briant, C. L. (2002). Crack tip deformation fields in ductile single crystals. Acta Materialia, 50(9), 2367-2380.

Kysar, J. W., Gan, Y. X., \& Mendez-Arzuza, G. (2005). Cylindrical void in a rigid-ideally plastic single crystal i: Anisotropic slip line theory solution for face-centered cubic crystals. International Journal of Plasticity, 21 (8), 1481-1520.

Kysar, J. W., Gan, Y. X., Morse, T. L., Chen, X., \& Jones, M. E. (2007). High strain gradient plasticity associated with wedge indentation into face-centered cubic single crystals: Geometrically necessary dislocation densities. Journal of the Mechanics and Physics of Solids, 55(7), 1554-1573.

Kysar, J. W., Saito, Y., Oztop, M. S., Lee, D., \& Huh, W. (2010). Experimental lower bounds on geometrically necessary dislocation density. International Journal of Plasticity, 26(8), 1097-1123.

Larson, B. C., El-Azab, A., Yang, W., Tischler, J. Z., Liu, W., \& Ice, G. E. (2007). Experimental characterization of the mesoscale dislocation density tensor. Philosophical Magazine, 87(8-9), 1327-1347.

Larson, B. C., Yang, W., Tischler, J. Z., Ice, G. E., Budai, J. D., Liu, W., \& Weiland, H. (2004). Micron-resolution 3-D measurement of local orientations near a grain-boundary in plane-strained aluminum using x-ray microbeams. International Journal of Plasticity, $20(3), 543-560$.

Maurice, C., Driver, J. H., \& Fortunier, R. (2012). On solving the orientation gradient dependency of high angular resolution ebsd. Ultramicroscopy, 113, 171-181.

Nye, J. F. (1953). Some geometrical relations in dislocation solids. Acta Metallurgica, 1(2), $153-162$.

Ohashi, T., Barabash, R. I., Pang, J. W. L., Ice, G. E., \& Barabash, O. M. (2009). X-ray microdiffraction and strain gradient crystal plasticity studies of geometrically necessary dislocations near a Ni bicrystal grain boundary. International Journal of Plasticity, 25(5), 920-941.

Pang, J. W. L., Liu, W., D., B. J., \& Ice, G. E. (2014). Inhomogeneous deformation behavior in intercrystalline regions in polycrystalline ni. Acta Materialia, 65, 393-399.

Pantleon, W. (2008). Resolving the geometrically necessary dislocation content by conventional electron backscattering diffraction. Scripta Materialia, 58(11), 994-997.

Peirce, D., Asaro, R., \& Needleman, A. (1983). Material rate dependence and localised deformation in crystalline solids. Acta Metallurgica, 31 (12), 1951-1976.

Rester, M., Motz, C., \& Pippan, R. (2007). Microstructural investigation of the volume beneath nanoindentations in copper. Acta Materialia, 55(19), 6427-6435. 
Rice, J. R. (1987). Tensile crack tip fields in elastic-ideally plastic crystals. Mechanics of Materials, 6(4), 317-335.

Ruggles, T. J., \& Fullwood, D. T. (2013). Estimations of bulk geometrically necessary dislocation density using high resolution ebsd. Ultramicroscopy, 133, 8-15.

Sun, S., Adams, B. L., \& King, W. E. (2000). Observations of lattice curvature near the interface of a deformed aluminium bicrystal. Philosophical Magazine A, 80(1), 9-25.

Wu, T. Y., Bassani, J. L., \& C., L. (1991). Latent hardening in single crystals I: Theory and experiments. Proceedings of the Royal Society of London Series A, 435(1893), 1-19.

Zaafarani, N., Raabe, D., Singh, R. N., Roters, F., \& Zaefferer, S. (2006). Three-dimensional investigation of the texture and microstructure below a nanoindent in a $\mathrm{Cu}$ single crystal using 3D EBSD and crystal plasticity finite element simulations. Acta Materialia, 54(7), 1863-1876. 